ACCEPTED MANUSCRIPT

\title{
Inverse Compton radiation: a novel X-ray source for K-edge subtraction angiography?
}

To cite this article before publication: Gianfranco Paternò et al 2019 Phys. Med. Biol. in press https://doi.org/10.1088/1361-6560/ab325c

\section{Manuscript version: Accepted Manuscript}

Accepted Manuscript is "the version of the article accepted for publication including all changes made as a result of the peer review process, and which may also include the addition to the article by IOP Publishing of a header, an article ID, a cover sheet and/or an 'Accepted

Manuscript' watermark, but excluding any other editing, typesetting or other changes made by IOP Publishing and/or its licensors"

This Accepted Manuscript is $\odot 2019$ Institute of Physics and Engineering in Medicine.

During the embargo period (the 12 month period from the publication of the Version of Record of this article), the Accepted Manuscript is fully protected by copyright and cannot be reused or reposted elsewhere.

As the Version of Record of this article is going to be / has been published on a subscription basis, this Accepted Manuscript is available for reuse under a CC BY-NC-ND 3.0 licence after the 12 month embargo period.

After the embargo period, everyone is permitted to use copy and redistribute this article for non-commercial purposes only, provided that they adhere to all the terms of the licence https://creativecommons.org/licences/by-nc-nd/3.0

Although reasonable endeavours have been taken to obtain all necessary permissions from third parties to include their copyrighted content within this article, their full citation and copyright line may not be present in this Accepted Manuscript version. Before using any content from this article, please refer to the Version of Record on IOPscience once published for full citation and copyright details, as permissions will likely be required. All third party content is fully copyright protected, unless specifically stated otherwise in the figure caption in the Version of Record.

View the article online for updates and enhancements. 


\title{
Inverse Compton radiation: a novel X-ray source for K-edge subtraction angiography?
}

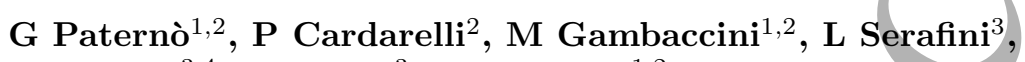 \\ V Petrillo ${ }^{3,4}$, I Drebot ${ }^{3}$ and A Taibi ${ }^{1,2}$ \\ 1 Dipartimento di Fisica e Scienze della Terra, Università di Ferrara, Via G.
} Saragat 1, 44122 Ferrara, Italy

2 INFN - Sez. Ferrara, Via G. Saragat 1, 44122 Ferrara, Italy

3 INFN - Sez. Milano, via Celoria 16, 20133 Milano, Italy

${ }^{4}$ Dipartimento di Fisica, Università di Milano, via Celoria 16, 20133 Milano, Italy

E-mail: cardarelli@fe.infn.it

July 2019

Abstract. Coronary angiography is clinically used worldwide to diagnose diseases of coronary arteries. Despite its effectiveness, this technique is quite invasive and it is associated to significant risks due to the arterial catheterisation needed to inject the contrast agent. A valid alternative is using the K-edge subtraction method, which is based on the subtraction of two images acquired at energies bracketing the K-edge of the contrast element. The enhanced sensitivity of K-edge subtraction allows the intravenous injection of the contrast agent, thus reducing the risks of catheterisation. This technique can be effectively implemented by using intense and quasi-monochromatic X-ray beams. Synchrotron radiation has been proven to work well for this purpose, but its cost and size prevent a widespread clinical application. Inverse Compton sources are among the most promising innovative sources of intense and quasi-monochromatic X-rays. These sources are intrinsically more compact than those based on synchrotron radiation. In this work, the potential application of inverse Compton radiation to K-edge subtraction angiography is investigated. To this purpose, after a short review of the physics behind the inverse Compton process, an analytical framework is described. The proposed model is based on the application of the K-edge subtraction algorithm to calculate the $S N R$ of details inside a suitable mathematical phantom. That allowed us to identify the characteristics of an inverse Compton source required for K-edge subtraction imaging. In particular, it was estimated that a photon fluence of $10^{8} \mathrm{ph} \mathrm{mm}^{-2}$ is necessary to detect signals of cliniçal interest. Novel sources based on inverse Compton promise to achieve this requirement with an acquisition time of few hundreds of ms. This feature, together with compactness, broad two-dimensional radiation field, absence of harmonic contamination and the ability to deliver high photon fluxes also at high energies, makes this kind of sources promising for K-edge subtraction angiography and other diagnostic applications.

Keywords: Inverse Compton, monochromatic source, K-edge subtraction, Dual-energy, Angiography 


\section{Introduction}

Coronary angiography is the standard of reference for the X-ray imaging of coronary anatomy and for establishing the presence and extent of coronary artery diseases (Tavakol et al. 2012). This imaging technique reveals extent, location, and severity of coronary obstructive lesions, which are effective predictors of outcome, and identifies high-risk patients who may benefit from revascularisation. It relies on the injection of a contrast agent based on iodine inside the coronary circulation, by means of artery catheterisation. X-ray imaging of the heart before and after the injection of contrast medium allows to subtract the background signal due to tissues different from iodine. This technique is quite invasive, with a morbidity just below $2 \%$ and patient mortality slightly less than $0.08 \%$, mainly related to the arterial catheterisation (Tavakol et al. 2012). Also, complications are frequently associated with the high amount of iodine-based contrast medium that are injected during the procedure, leading to allergic reactions or nephrotoxic effects. The number of patients harmed by these adverse effects can be lowered if a good diagnostic image quality is achieved with a reduced amount of contrast medium being injected. Moreover, an intravenous injection would eliminate the risks due to arterial catheterisation.

An alternative technique is based on K-edge subtraction (KES), which takes advantage of the sharp rise of the photo-electric absorption coefficient of the contrast agent injected in the tissues to be visualised. Two images are acquired at energies bracketing the K-edge of the element, ideally with monochromatic X-ray beams. The logarithmic subtraction of the low- and high-energy images enhances the structures filled with the contrast agent, while the signal produced by the surrounding tissues is nearly cancelled since their attenuation coefficients are almost identical at the two energies (Jacobson 1953, Sarnelli et al. 2004). The enhancement in sensitivity allowed by this imaging technique makes possible the intravenous injection of the iodine and reduces the post-interventional morbidity due to the arterial catheterisation performed in conventional coronary angiography (Johnson et al. 1993, Bertrand et al. 2005, Dix et al. 2003).

Implementation of KES is possible with two X-ray beams having different mean energy and a narrow bandwidth. Many solutions have been experimented using suitable X-ray tube filtration (Mistretta et al. 1973), X-ray fluorescence (Rutt et al. 1983) and quasi-monochromatic beams obtained through diffraction from a crystal (Sarnelli et al. 2004). Each of the aforementioned methods could not provide a sufficient intensity and/or an appropriate bandwidth for an effective clinical application. To date, synchrotron radiation has been the only source providing sufficiently intense monochromatic X-ray beams. An alternative solution proposed for KES implementation with polychromatic X-ray beams relies on the energy discrimination obtainable by using multi-threshold photon-counting detectors (see for example the work of (Roessl \& Proksa 2007)). However, the application of this technique is not straightforward due to the typical energy resolution (1-2 keV) achievable on the threshold (Fredenberg 2018), which limits the energy discrimination accuracy,

The K-edge subtraction angiography has been fully tested by using synchrotron radiation and several research protocols have been developed to evaluate the performance of this method in comparison with the conventional one. At DESY in Hamburg, Germany (Dix et al. 2003), two hundred and thirty patients were investigated with the NIKOS IV system (Dix et al. 1996, Dill et al. 1998). The study 
demonstrated the feasibility and safety of the method and its ability to provide detailed images of coronary artery segments and very high specificity for severe stenoses. At the European Synchrotron Radiation Facility (ESRF), a beamline is dedicated to medical applications of synchrotron radiation (Elleaume et al. 1999, Dabin et al. 2001) and results of the research protocol for KES angiography have been reported in the literature (Elleaume et al. 2000). Several patients were imaged and a good agreement was achieved between synchrotron angiography and the conventional angiography procedure adopted in hospital, in terms of stenosis diagnosis. These tests have shown that synchrotron angiography was capable of producing clinical quality images in a significant portion of the coronary anatomy (Thomlinson et al. 2018). The major limitations of the technique are the unevaluable segments, which depend on the projection angle and the superposition with the left ventricle, the aorta or the pulmonary veins. Others problems arise from a low signal-to-noise ratio in overweight patients. On the other hand, these studies proved the advantages of the technique in the assessment of stents, early and late post-operative evaluation of the effects of by-pass surgery and follow up of patients at high risk of in-stent restenosis (Bertrand et al. 2005, Dix et al. 2003). Another advantage of venous catheterisation is that the coronary arteries are not artificially pressurised and the resulting images are therefore acquired in a true physiological state.

Synchrotron radiation is the most suitable source for any application requiring a high-brilliance monochromatic X-ray beam. On the other hand, the economic effort required to build, commission and operate a synchrotron facility is beyond the means of any small research institution or clinic. The limited accessibility to monochromatic intense X-ray sources prevents the transfer of KES angiography, as well as many other diagnostic techniques, to clinical routine. A compact and affordable source, providing a beam with synchrotron-like features, would open the way to a wide range of innovative clinical applications and medical research, both in diagnosis and therapy.

Inverse Compton sources (ICS), based on the interaction of an intense laser beam and a bright beam of relativistic electrons, are among the most promising innovative sources of (quasi-)monochromatic $\mathrm{X}$ and gamma radiation. For this reason, many laboratories worldwide are operating or proposing scientific programs aimed to develop and commission facilities based on inverse Compton scattering. Various technologies have been proposed and implemented to provide radiation beams having different energy ranges, spectra and features able to fulfil the requirements of different applications. Furthermore, within the X-ray energy range of few tens of $\mathrm{keV}$, various research groups have demonstrated its feasibility and performed preliminary imaging experiments at available ICSs, such as SPARC-LAB (INFN-LNF, Frascati, Italy) (Vaccarezza et al. 2016), COBALD (STFC, Daresbury, UK) (Priebe et al. 2010), LCS (AIST, Japan) (Kuroda et al. 2011), TTX (Tshingua University, Bejing, China) (Tang et al. 2009). In Europe two user facilities are currently under commissioning, with expected beginning of operation in 2019: STAR (University of Calabria, Cosenza, Italy) (Bacci et al. 2014) and ThomX (LAL, Orsay, France) (Variola 2011). Also, an operative user facility was installed in 2015 at Technical University of Munich, Germany: Munich Compact Light Source (MuCLS) (Eggl et al. 2016). Even if ICSs available nowadays are not table-top sources, this technology represents a huge step forward in the direction of filling the gap between synchrotron facilities and currently available laboratory size sources. In fact, the electron energy required to provide the same X-ray energy is at least one order of magnitude less for ICS (10-100 MeV) than for synchrotron radiation $(1-10 \mathrm{GeV})$. Therefore, the scale of the accelerator hardware,

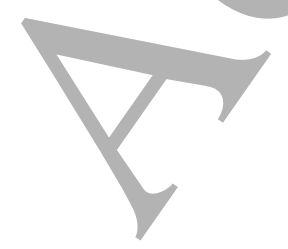


shielding, operational cost and dedicated staff are significantly reduced. Furthermore, inverse Compton beams have a higher divergence with respect to synchrotron radiation (thousands against hundreds of $\mu \mathrm{rad}$ ) and this allows to obtain a radiation field with a size of the order of $10 \times 10 \mathrm{~cm}^{2}$ at less than $100 \mathrm{~m}$ of distance from the interaction point. This feature is very useful in most of medical imaging applications, where the broadness and uniformity of the radiation field are stringent requirements. In many cases, the broad two-dimensional field obtainable allows to acquire radiographs without scanning the patient or the beam.

A recent review concerning the development of K-edge subtraction technique and technology applied to bio-medical imaging clearly states that, with the advent of new X-ray sources, such as compact Compton sources, KES imaging research and potential clinical applications will continue to be important areas of biomedical research (Thomlinson et al. 2018). KES implementation is possible when two X-ray beams having different mean energy are available simultaneously or if the energy of a single X-ray beam can be switched swiftly. Various technological solutions aimed at providing a dual-energy ICS having a time structure compatible with an imaging task have been proposed (Drebot et al. 2017, Drebot et al. 2018a). Recently, a proposal for a Multidisciplinary Advanced Research Infrastructure with X-rays (MariX) has been supported by University of Milan and the Italian Institute for Nuclear Physics (INFN) (Serafini et al. 2018, Serafini et al. 2019). This facility is expected to include an ICS with performance comparable to those of modern synchrotron light sources, combined with a dual-colour mode that enables the fast switch (few hundreds of ms) between two different values of mean energy of the X-ray beam. The advent of these technologies will make possible, in the near future, the realistic application of ICS to Kedge subtraction technique in the field of bio-medical imaging. Experiments on X-ray imaging with ICS involving K-edge absorption discontinuity have been reported, such as K-edge filtering to measure X-ray emission spectrum (Golosio et al. 2012, Williams et al. 2009), imaging with (quasi-)monochromatic beam at an energy above K-edge (Yamada et al. 2009, Carroll 2003, Eggl et al. 2017, Kuroda 2014) and subtraction imaging with quasi-monochromatic beam and subsequent added filtration of the same material used as contrast medium (Kulpe et al. 2018). However, to our knowledge, dual-energy KES experiments with ICS have never been carried out so far, due to the difficult implementation of a fast dual-energy switch.

The aim of this work is to investigate the potential of KES imaging using the quasi-monochromatic X-ray beams obtained from inverse Compton interaction. Due to the physics behind this process, the characteristics of the produced X-rays such as energy bandwidth, spatial distribution and flux are all interconnected and depend on the features of the interacting electron and laser beams. Therefore, a short review of inverse Compton is given to provide the theoretical background necessary to analyse the parameters that affect the outcome of the image formation process. A quantitative framework was developed to identify the features of an ICS that meet the requirements of a possible medical application of KES imaging and is here described. Coronary angiography was selected as imaging task, being one of the most demanding in terms of X-ray beam intensities, due to the significant attenuation of surrounding tissues in the chest and the low signal provided by the iodinated coronary arteries. Results in terms of fluence required to image most of coronary arteries and their possible stenoses with adequate $S N R$ are provided. A discussion on the performance of an ICS having characteristics comparable with those of the ICS currently under commissioning or in proposal stage is also presented. 


\section{Theoretical background}

\subsection{Inverse Compton sources}

Inverse Compton sources, sometimes also called Thomson Backscattering Sources (TBS) or Laser Compton Sources (LCS), are based on the interaction of a laser beam with a relativistic electron beam. An exhaustive treatment of the inverse Compton scattering, in both linear and non-linear regime, can be found in specialised and review articles (see, for instance, (Esarey et al. 1993, Brown \& Hartemann 2004, Sun \& Wu 2011, Petrillo et al. 2012, Krafft et al. 2016)). Here we briefly describe the process, focusing on the features that are important for the discussion about potential use of the emitted radiation in radiology and, in particular, in KES imaging.

Figure 1. Sketch of the inverse Compton scattering process.

Consider an electron and a laser photon interacting with the geometry described in figure 1. The electron has an energy $E_{e}$ and is moving along the $\mathrm{z}$ direction in the laboratory frame. The lasser photon has an energy $E_{L}$ and impinges with a polar angle $\theta_{i}$ with respect to the electron propagation direction. The collision occurs at the origin of the coordinate system. After the collision, the photon is scattered into the direction defined by polar angle $\theta$ and azimuthal angle $\phi$. The energy of the scattered photon $E$ can be calculated from conservation of the 4-momenta before and after the scattering event. As a result of the interaction, $E$ is related to the polar scattering angle. For a small polar scattering angle $(\theta<<1)$ and an ultra-relativistic electron (Lorentz factor $\gamma=E_{e} /\left(m c^{2}\right)>>1$, being $m$ the electron mass and $c$ speed of light in vacuum) it holds

$$
E=\frac{4 \gamma^{2} E_{L} \cos ^{2} \alpha / 2}{1+\gamma^{2} \theta^{2}+R},
$$

where $R=4 \gamma^{2} E_{L} / E_{e} \cos ^{2} \alpha / 2$ and $\alpha=\pi-\theta_{i}$ is the collision angle. The energy of the scattered photon is independent from the azimuthal angle $\phi$, while it has a Lorentzian dependence on the polar angle $\theta$. The presence of $R$ in the denominator is due to the electron recoil and has the effect of slightly reducing the energy of the scattered photon. In many practical cases, the recoil is small and $R$ can be neglected (Thomson regime). For backscattering events, namely when the photon is scattered in the same direction of the electron $(\theta=0), E$ reaches the maximum value

$$
E_{\text {max }} \simeq 4 \gamma^{2} E_{L} \cos ^{2} \alpha / 2 .
$$

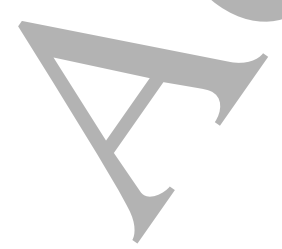


In the case of a head-on interaction $(\alpha=0)$, the laser photon energy is boosted to a factor of about $4 \gamma^{2}$ for a backscattering event $(\theta=0)$. Therefore, the Compton scattering of photons with relativistic electrons can be used to produce high-energy photons, i.e., X-rays and gamma photons. It is worth noting that, if optical photons with a typical energy $E_{L}=1 \mathrm{eV}$ are considered, an accelerated electron beam with $E_{e}=50 \mathrm{MeV}(\gamma=97.85)$ will generate X-rays with a maximum energy of $38.3 \mathrm{keV}$. To obtain X-rays in this energy range with a typical synchrotron insertion-device (bending magnet or wiggler), energies in the range 1-10 GeV are required for the electron beam. For this reason, this kind of source is usually considered as a promising compact alternative to synchrotron.

The energy spectrum of an ICS follows the classical Compton distribution in the range from $E_{L}$ to $E_{\max }$. About half of the photons are emitted within a cone with aperture $1 / \gamma$ (Krafft \& Priebe 2010). The characteristic correlation between energy and scattering angle can be exploited to adjust the energy bandwidth of the photon beam through a proper collimation system that filters out the radiation emitted at larger polar angles (Paternò et al. 2017). For a small normalised acceptance angle $\psi=\gamma \theta_{\max } / \sqrt{1+R}$, which is simply $\psi=\gamma \theta_{\max }$ for $R<<1$, the relative energy bandwidth $B W_{\psi}$ scales approximately with $\psi^{2}$ (Petrillo et al. 2012).

When bunches of electrons collide with laser pulses, the number of scattered photons $N_{\psi}$ within a normalised acceptance angle $\psi$ can be calculated by integrating the differential Compton cross-section over the solid angle with aperture half-angle $\theta_{\max }$ and multiplying it by the collision luminosity*. Practical scaling laws obtained following this approach can be found in the literature (Petrillo et al. 2012, Curatolo et al. 2017). Here, it is worth noting that $N_{\psi}$ is proportional to the charge of the electron bunch, the number of photons in a laser pulse and the frequency of collision, while it depends inversely on the cross-section of the colliding beams and scales roughly with $\psi^{2}$ for small values of $\psi$. Moreover, $N_{\psi}$ decreases if the size of the colliding beams and the collision angle $\alpha$ increase. The emission spot also depends on the size of the colliding beams. Therefore, the colliding beams have to be as small as possible in order to maximise the number of photons within the collimation angle and have an emission spot of small size.

It is also worth noting that the previous treatment holds in the low laser intensity regime (laser strength parameter $\sharp a_{0}<<1$ ). As the laser intensity gets higher, nonlinear effects arise. These affect the spectral distribution of the scattered photons, with the harmonics contribution becoming more significant as the laser strength increases. The relative amplitude of the higher harmonics with respect to the fundamental scales as the square of $a_{0}$ (Tomassini et al. 2008, Maroli et al. 2013). Hereinafter, we will consider ICSs operating in the low laser-intensity regime, for which non-linear effects and higher harmonics contribution are negligible.

Figure 2 shows the typical behaviour of $B W_{\psi}$ and $N_{\psi}$ as a function of the acceptance angle $\theta_{\max }$, for an ICS emitting photons with maximum energy of 34.3

* Luminosity describes the ability of a particle accelerator to produce the required rate of interactions. Indeed, it is the proportionality factor between the number of events per second and the cross section of a physical process of interest. Examples of luminosity calculation in various cases can be found in the literature (Patrignani et al. 2016, Herr \& Muratori 2006).

\# The laser strength parameter $a_{0}$ is a dimensionless number defined as the modulus of the normalized vector potential of the electromagnetic field associated to the laser. It can be written as: $a_{0}=e E_{0} / m_{E} c \omega_{L}=8.6 \lambda_{L}(\mu \mathrm{m}) \sqrt{I\left(\mathrm{~W} / \mathrm{cm}^{2}\right)}$ where $E_{0}$ and $\omega_{L}$ are the maximum value and the pulsation of the laser electric field, $c$ the speed of light in vacuum, $m_{e}$ the electron mass, $\lambda_{L}$ the laser wavelength, $I$ the laser intensity. 
$\mathrm{keV}$. The smaller the acceptance angle, the smaller the relative energy bandwidth and

a)

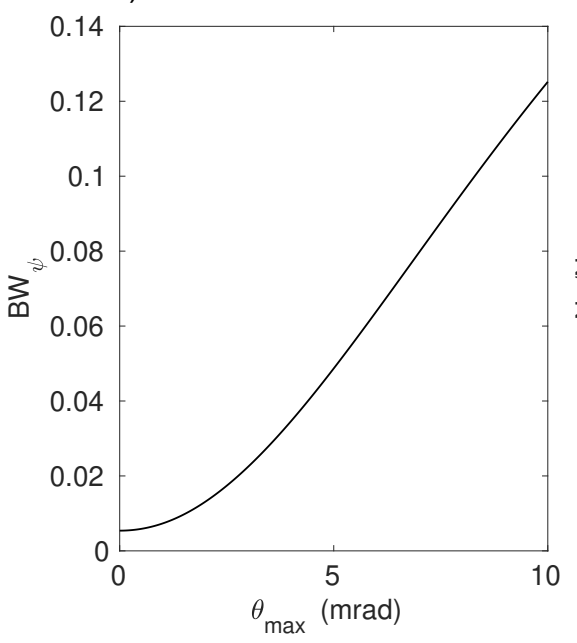

b)

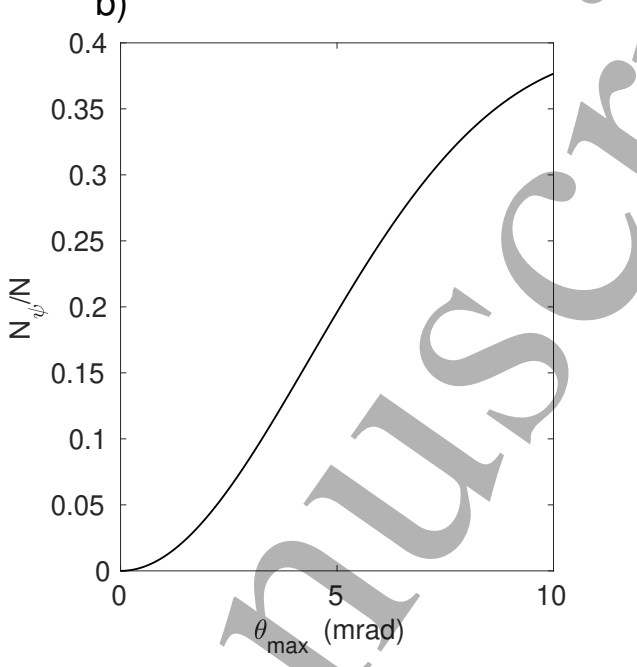

Figure 2. a) Relative energy bandwidth $B W_{\psi}$ and b) number of photons emitted within a given acceptance angle $N_{\psi}$ (normalised with respect to the total emitted photons $N$ ) as a function of the acceptance angle $\theta_{\max }$ for a generic ICS emitting photons with maximum energy of $34.3 \mathrm{keV} . E_{L}=1.2 \mathrm{eV}$ and $E_{e}=43.2 \mathrm{MeV}(\gamma$ $=84.5)$.

the number of emitted photons within the acceptance angle. Indeed, as mentioned above, the dependence of $B W_{\psi}$ and $N_{\psi}$ on $\theta_{\max }$ is roughly quadratic for small value of $\theta_{\max }$, while it is apparent that this is no longer valid for large acceptance angles. It is also worth noting that there is a lower limit to the achievable monochromaticity of the scattered photon beam. Indeed, apart from the acceptance angle, the energy spread of the radiation depends on the energy spread of the colliding beams, on the electron beam emittance and on the laser parameters (Curatolo et al. 2017, Ranjan et al. 2018). The choice of a collimation angle $\theta_{\max }$ smaller than a critical value, which depends on the specific ICS, causes only a decrease of the number of emitted photons. This is not a severe limitation for medical applications, since the energy bandwidth can reach values lower than $1 \%$. If a smaller bandwidth is required for a particular application, such as X-ray absorption near edge structure (XANES) spectroscopy, a further monochromatisation technique can be applied in addition to collimation. In particular, a crystal monochromator can be exploited, so as to reduce the X-ray beam energy bandwidth through Bragg diffraction at the expense of photon flux. For the purpose of this work, we do not consider any additional monochromator.

It is important to take into account that, for each value of the scattering angle $\theta$, the scattered photons feature a distribution of energies whose mean value is given by equation (1) and spread depends on the beam parameters and varies with $\theta$. The local energy bandwidth, namely the bandwidth of the spectrum at a fixed observation direction, increases as $\theta$ increases, although it is always smaller than the bandwidth of the whole collimated spectrum $B W_{\psi}$. The local bandwidth approaches $B W_{\psi}$ for collimation angle close the critical one. A description of the energy distribution at a fixed observation angle and of a method, based on a K-edge filtering technique, to

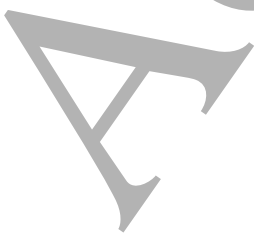


measure the local spectrum can be found in the literature (Golosio et al. 2012).

The collimation angle defines the maximum divergence of the emitted photon beam. Since the cross-sections of the colliding beams are small, the emission spot of an ICS is typically very small (in the range between 10 and $100 \mu \mathrm{m}$ ) and the source can be considered as point-like. Therefore, the photon beam at a distance $d$ from the interaction point (IP) is a circle with diameter $D$ given by

$$
D=2 d \tan \theta_{\text {max }} \simeq 2 d \theta_{\text {max }},
$$

being $\theta_{\max }<<1$. If a collimation angle close to the critical one is set, the radiation field has uniform intensity and energy distribution. Otherwise a gradient in the intensity and spectral distribution would be present, with the outermost part of the radiation field being less intense and involving less energetic photons with respect to central part. This aspect is very important for medical diagnostic applications where a broad uniform radiation field is desirable.

\subsection{Dual-energy ICSs}

In order to implement a dual-energy K-edge subtraction imaging system, it is necessary to provide an X-ray beam that can switch the energy between two values bracketing the K-edge energy of interest. From equation (2), it comes out that it is possible to act on three parameters to adjust the maximum energy of the radiation emitted: the electron energy, the laser wavelength and the collision angle. As a result of the collimation, the energy of the beam extends from $E_{\min }=E\left(\theta_{\max }\right)$ to $E_{\max }=E(0)$, therefore, a change in $E_{\max }$ causes a corresponding change in the mean energy of the quasi-monochromatic X-ray beam emitted. In most of the ICS in operation or being realised, the X-ray beam energy tuning is obtained by varying the electron beam energy, while the laser wavelength and the collision angle are fixed. A modification of electron energy is possible only on a time-scale longer than that required for $\mathrm{X}$ ray medical imaging which, depending on the specific application, is within $0.1-10$ seconds. Therefore, in order to provide a dual-colour mode with a time structure compatible with an imaging task, the most promising solutions are those in which the $\mathrm{X}$-ray energy tuning is made by quickly modifying the collision angle. The possibility of exploiting the interaction of the electron beam with two light pulses colliding with the electrons at a different angle has been recently proposed and investigated (Drebot et al. 2017, Drebot et al. 2018a). The two laser pulses can come from the same laser or from two different lasers. Figure 3.a) shows the dependence of the maximum energy of the beam on the collision angle $\alpha$, for a typical ICS in the energy range of interest for KES imaging. A relative separation of about $7 \%$ for the maximum energy of the two nearly-monochromatic X-ray beams can be obtained if, for instance, $\alpha$ is set to $0^{\circ}$ and $30^{\circ}$ respectively, for the interaction of the two laser pulses with the electron beam. It is worth noting that the collimation angle $\theta_{\max }$ has to be chosen properly to obtain a satisfactory separation between the two X-ray peaks.

Also, due to the dependence of $N_{\psi}$ on $\alpha$ (see Figure 3.b)), the HE and LE Xray beams feature different intensities unless appropriate actions are taken. To this purpose, it is possible to use two lasers of different intensity or, in alternative, the two laser beams could be focused to a different size. In a recent work, the authors have proposed the scheme of a Fabry-Pérot optical cavity that allow to vary the laser beam waist independently from the cavity round trip frequency (which must be matched to that of the electron beam) (Drebot et al. 2018b). A dual-colour ICS could be then 
a)

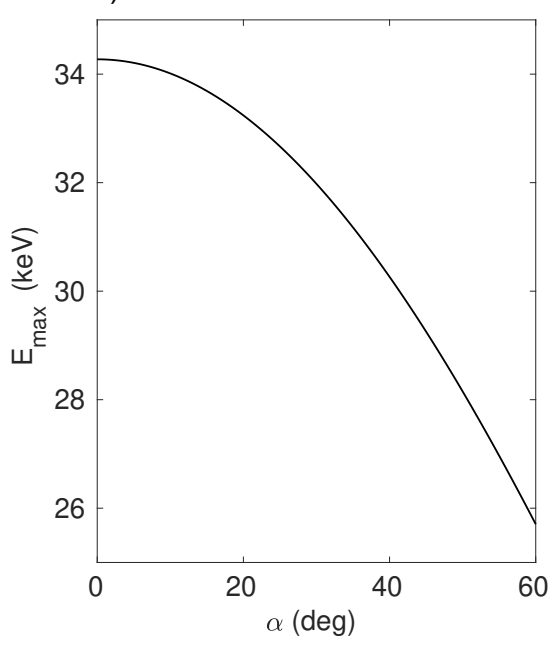

b)

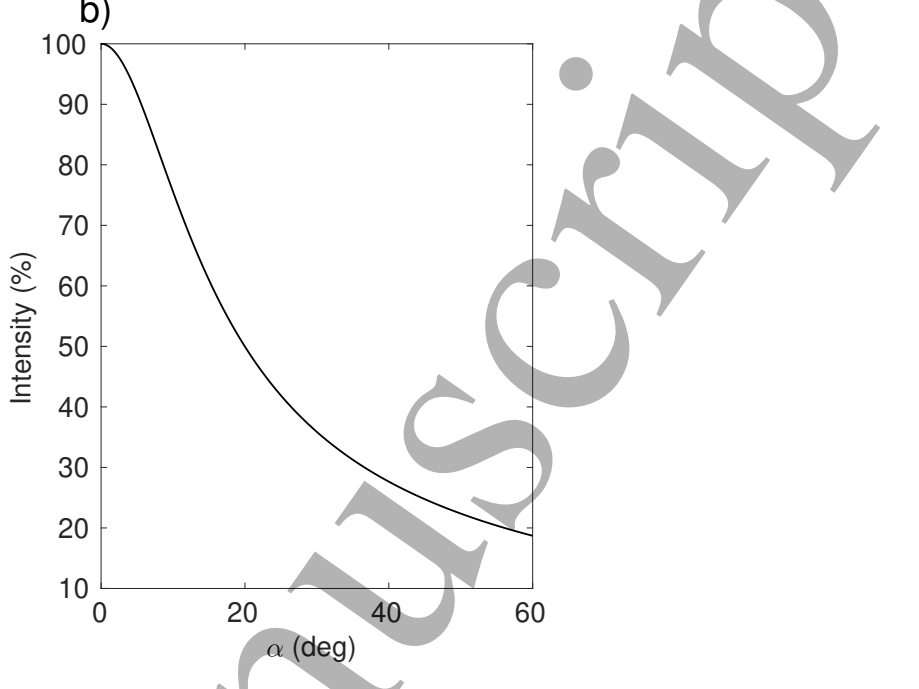

Figure 3. a) Maximum energy and b) intensity of the emitted beam as a function of the collision angle $\alpha$ for $\gamma=84.5$ and $E_{L}=1.2 \mathrm{eV}$.

developed, as foreseen for MariX, by exploiting two of these cavities, each one focusing the laser beam differently, according to the collision angle. For this reason, we adopted this method in our calculations.

\subsection{Mathematical formalism for KES radiography}

The KES algorithm is based on the dual-energy method (Alvarez \& Macovski 1976, Lehmann et al. 1981), which consists in decomposing the total attenuation of two nearly-monoenergetic X-ray beams crossing a sample in the contribution of two proper basis materials. If a contrast element and a soft tissue are chosen as basis materials, the negative logarithm of the attenuation $C^{ \pm}$produced by the sample for two monochromatic beams at energies $E^{+}$and $E^{-}$bracketing the K-edge of the contrast element is

$$
C^{ \pm}=\left[\frac{\mu^{ \pm}}{\rho}\right]_{X}(\rho t)_{X}+\left[\frac{\mu^{ \pm}}{\rho}\right]_{T}(\rho t)_{T},
$$

where $X$ indicates a generic contrast element, such as iodine (I) or gadolinium (Gd), $T$ the soft tissue, $\mu / \rho$ is the mass attenuation coefficient, $\rho$ the density, and $t$ the material thickness. The superscripts \pm indicate the two energies $E^{+}$and $E^{-}$. Neglecting the scattering contribution and the electronic noise, the number of photons $N^{ \pm}$acquired by each pixel of a detector/positioned just behind the sample can be related to the value of $C^{ \pm}$as follows

$$
C^{ \pm}=\ln \frac{\varepsilon F \phi_{0}^{ \pm}}{N^{ \pm}},
$$

where $\phi_{0}^{ \pm}$is the incident photon fluence at energies $E^{+}$and $E^{-}, \varepsilon$ the detector efficiency (assumed identical for both energies) and $F$ the area of the detector pixels. 
The mass thicknesses (surface densities) $(\rho t)_{X}$ and $(\rho t)_{T}$ can be calculated by solving the two independent equations (one for each energy) obtained from (4). They result

$$
\begin{aligned}
& (\rho t)_{X}=\frac{\left[\frac{\mu^{-}}{\rho}\right]_{T} C^{+}-\left[\frac{\mu^{+}}{\rho}\right]_{T} C^{-}}{K_{0}}, \\
& (\rho t)_{T}=\frac{\left[\frac{\mu^{+}}{\rho}\right]_{X} C^{-}-\left[\frac{\mu^{-}}{\rho}\right]_{X} C^{+}}{K_{0}},
\end{aligned}
$$

where

$$
K_{0}=\left[\frac{\mu^{-}}{\rho}\right]_{T}\left[\frac{\mu^{+}}{\rho}\right]_{X}-\left[\frac{\mu^{+}}{\rho}\right]_{T}\left[\frac{\mu^{-}}{\rho}\right]_{X} .
$$

(1)

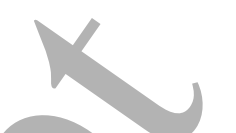

Equations (6) and (7) describe the 'contrast' and 'soft tissue'/image, respectively. In the former, the contrast element is virtually isolated from the background; in the latter, the mass thickness of all materials traversed by the beams (with the exception of the contrast element) are expressed in terms of the mass thickness of the soft tissue. It is worth noting that dual-energy equations are easily solved for narrow-band x-ray beams (Thomlinson et al. 2018) but usually require experimental calibration of the system with two basis materials for polychromatic x-ray spectra. Beam hardening corrections make use of non-linear models, as presented in the initial works of Macovski and colleagues (Alvarez \& Macovski 1976, Lehmann et al. 1981) and discussed very recently by our research group (Contillo et al. 2015). For quasi-monochromatic X-ray spectra, the beam quality is not changed by the transmission through the patient and the relationship between the log-measurement of attenuation and mass thicknesses is still linear. In particular, the difference in the mean energy between the input and the output spectra is within $0.5 \%$ for quasi-monochromatic X-ray beams with few percent energy bandwidth traversing $19 \mathrm{~cm}$ of soft tissue and $1 \mathrm{~cm}$ of bone (i.e., mimicking a typical chest). For this reason, no hardening correction is required in this case. The assumption of monochromaticity in the calculation is a valid approximation, provided that the peak separation and the bandwidth are such as to avoid that a significant portion of each X-ray beam spectrum crosses the contrast agent K-edge or overlaps with the spectrum of the other beam.

The proposed approach holds for a generic sample in which, apart from the contrast medium, there is no material that has a mass attenuation coefficient with a slope significantly different fromm that of the soft tissue in the energy range of interest for KES imaging, such as bone. If this is not the case, the decomposition of the total absorption should, in principle, explicitly include the contribution of this material and an exposure to three beams with three different energies is required to solve the equations. This is the case of coronary angiography, in which the presence of rib bone should be taken into account (Rutt et al. 1983). However, the dual-energy approach can still be used. Indeed, in this case, the signal due to the contrast agent flowing in the coronary arteries competes with a residual signal due to an incomplete cancellation of the bone structures in the contrast image, but the relative weight of this contribution can be reduced with a proper choice of the energy separation $\Delta E$ between the two quasi-monochromatic beams. Typically, an energy separation smaller than $1 \mathrm{keV}$ is used in clinical protocols of KES imaging with synchrotron radiation (Thomlinson et al. 2018), since this value was previously calculated to guarantee a 
sufficient bone signal cancellation even with a ratio between bone mass thickness and contrast element mass thickness of about 2000 (Rutt et al. 1983). However, as it will be discussed later, in case of smaller values of the mass thickness ratio, a larger energy separation could be used to obtain an effective bone cancellation.

2.3.1. Contrast medium signal Equations (6) and (7) can be used to implement the KES algorithm for analysing two acquired images. If a sample with known composition is considered, the signal due to a blood vessel filled with contrast medium can be preventively evaluated. It is defined as the difference between the $(\rho t)_{X}$ value corresponding to the vessel and the background (Sarnelli et al. 2006).

The negative logarithm of attenuation for a region of the sample encompassing a vessel filled with a contrast medium solution is given by

$$
C^{ \pm}=\mu_{C M}^{ \pm} \cdot t_{C M}+\sum_{i \neq C M} \mu_{i}^{ \pm} t_{i}
$$

In Eq. 9 and throughout the whole manuscript, subscripts are used to indicate physical properties of the considered materials. These subscripts are: $X$ for a generic contrast element, $I$ for iodine, $T$ for soft tissue, $W$ for water and $B$ for bone. It is important to distinguish between the single contrast element, such as iodine or gadolinium, and the whole contrast medium $(C M)$, which is a compound or an aqueous solution of the contrast element. In this way, $\mu_{i}^{ \pm}$and $t_{i}$ indicate the linear attenuation coefficient and the thickness of a material, respectively. The notation $\sum_{i \neq C M}$ is used to indicate in a concise way that the sum extends to all materials apart from the contrast medium $(C M)$, despite the formal incorrectness. Furthermore, $t_{C} M$ is the thickness of a blood vessel filled with the contrast medium and $\mu_{C M}^{ \pm}$is the linear attention coefficient of the contrast medium itself. For an aqueous solution of iodine with concentration $c_{C M}$, the linear attenuation coefficient is

$$
\mu_{C M}^{ \pm}=\left(w_{W}\left(\mu^{ \pm} / \rho\right)_{W}+w_{I}\left(\mu^{ \pm} / \rho\right)_{I}\right) \rho_{C M}
$$

$w_{W}$ and $w_{I}$ being the mass fraction of water and iodine and $\rho_{C M}=c_{C M} / w_{I}$ the density of the solution. For the background, namely a region not perfused by the contrast agent, it holds

$$
C^{ \pm}=\sum_{i \neq C M} \mu_{i}^{ \pm} t_{i}
$$

The signal $S$ is calculated by substituting equations (9) and (11) in equation (6):

$$
S \simeq \frac{\left[\frac{\mu^{-}}{\rho}\right]_{T} \mu_{C M}^{+}-\left[\frac{\mu^{+}}{\rho}\right]_{T} \mu_{C M}^{-}}{K_{0}} t_{C M} .
$$

For small contrast medium concentrations $\left(<50 \mathrm{mg} / \mathrm{ml}\right.$, which means that $\rho_{C M} \simeq$ water density), it can be shown (Sarnelli et al. 2006) that the previous equation reduces to

$$
S \simeq c_{C M} \cdot t_{C M}
$$

namely, the signal depends on the vessel thickness and contrast medium concentration only and is independent from the energy of the two X-ray beams. 
2.3.2. Signal-to-noise ratio Under the assumption that the signal fluctuation are purely due to Poisson statistics and the electronic noise of the detection system is negligible, the noise for a background pixel in the contrast image, $\sigma_{P}$, can be calculated by substituting equation (5) in (6) and propagating the error. It results

$$
\sigma_{P}^{2}=\frac{1}{K_{0}^{2}}\left(\left[\frac{\mu^{-}}{\rho}\right]_{T}^{2} \frac{1}{N^{+}}+\left[\frac{\mu^{+}}{\rho}\right]_{T}^{2} \frac{1}{N^{-}}\right),
$$

where the counts $N^{ \pm}$related to the background pixel can be expressed, exploiting the notation introduced above, as

$$
N^{ \pm}=\varepsilon F \phi_{0}^{ \pm} \exp \left(-\sum_{i \neq C M} \mu_{i}^{ \pm} t_{i}\right)
$$

The signal-to-noise ratio for each pixel is $S N R_{p}=S / \sigma_{P}$.

For a region of area $A$ corresponding to $n$ pixels on the detector, the signal is given by equation (12), while the noise is $\sigma_{b g}=\sigma_{P} / \sqrt{n}$. Therefore, the signal-to-noise ratio is

$$
S N R=\frac{S}{\sigma_{b g}}=\frac{S}{\sigma_{P}} \sqrt{n}=S N R_{p} \sqrt{n}
$$

2.3.3. Incident photon fluence to obtain a given SNR Assuming that the photon beam impinging on the sample has the same intensity at the two energies, the photon fluence required to obtain a given $S N R$ for a region corresponding to $n$ pixels on the detector can be calculated by first substituting equation (15) in (14) and then substituting the obtained value of $\sigma_{P}$ in $S N R=S / \sigma_{P} \sqrt{n}$. It results

$$
\phi_{0}=\left[\frac{S N R}{S}\right]^{2} \frac{1}{n \varepsilon F K_{0}^{2}}\left(\left[\frac{\mu^{-}}{\rho}\right]_{T}^{2} \exp \left(\sum_{i \neq C M} \mu_{i}^{+} t_{i}\right)+\left[\frac{\mu^{+}}{\rho}\right]_{T}^{2} \exp \left(\sum_{i \neq C M} \mu_{i}^{-} t_{i}\right)\right) .
$$

This equation will be extensively used in the following, together with equation (12), to estimate the fluence required to image the details of a proper mathematical phantom.

2.3.4. Dose evaluation According to Sarnelli et al. (2006), the dose delivered to skin $D_{\text {skin }}$ is calculated as follows.

$$
D_{\text {skin }}=\phi_{0}\left\{E^{+}\left[\frac{\mu_{e n}^{+}}{\rho}\right]_{T}+E^{-}\left[\frac{\mu_{e n}^{-}}{\rho}\right]_{T}\right\},
$$

where $\left[\mu_{e n}^{ \pm} / \rho\right]_{T}$ are the mass energy-absorption coefficients of soft tissue at the mean energy of the two quasi-monochromatic beams.

\section{Materials and methods}

In order to eyaluate the effectiveness of KES imaging with X-ray beams obtained from an ICS, two quasi-monochromatic beams with energy bracketing the iodine K-edge were first considered. These beams were obtained from the Monte Carlo simulations previously performed for STAR, which is a medium-size ICS currently under commissioning at the University of Calabria (Bacci et al. 2014). The simulations were carried out through the Monte Carlo code CAIN (Chen et al. 1995). Starting from the features of the laser pulse and the phase space of the electron beam provided by the user, this code generates a complete phase space of the scattered X-ray photons. The

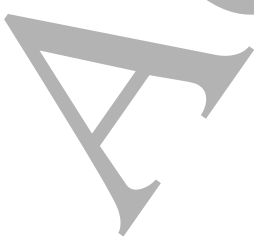


collision parameters and the details of the simulations can be found in the literature (Drebot et al. 2017). Figure 4 shows the normalised spectra of the high-energy (HE) and low-energy (LE) beams obtained from the simulations. A collimation angle of $1 \mathrm{mrad}$ was set in order to have an almost complete separation of the two peaks, which feature a mean energy of $31.7 \mathrm{keV}$ and $34.0 \mathrm{keV}$, respectively, and a relative energy bandwidth of $1.5 \%$. Indeed, in this case, only $4.2 \%$ of the photons of HE beam overlap with the LE spectrum. Figure 4 also shows the mass attenuation coefficient

of iodine, to highlight that simulated spectra are almost completely restricted to one side only of iodine K-edge. Indeed, in this case, only $5.5 \%$ of the photons of HE beam has an energy below the iodine K-edge, while there are almost no photons of the LE beam with energy above the iodine K-edge. This feature makes the simulated spectra good candidates for KES imaging with iodine. Furthermore, the radiation field obtained with this configuration is quite uniform, as shown in figure 5. Therefore, two monochromatic X-ray beams with the energy of the simulated peaks were used to calculate the $S N R$ ratio in a proper mathematical phantom. Such phantom mimics the attenuation of X-rays in a coronary angiography with an iodinated contrast medium. In the following subsection the details of this procedure are described. The results of this study provide the background for a discussion on the performance of an ICS in KES coronary/angiography.

\subsection{Study of the SNR in a phantom}

The KES imaging performance achievable with the proposed source was evaluated through analytical calculations carried out on the simple mathematical phantom shown in figure 6 . The phantom includes the main materials involved when a coronary artery has to be imaged. It consists of a bulk of soft tissue, defined according to ICRU report 44 (ICRU 1989) and having a thickness $t_{T}$. A vessel perfused with an iodinated contrast agent traverses the soft tissue undergoing stenosis in the central portion. The

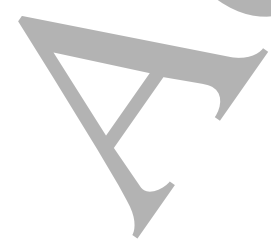


a)

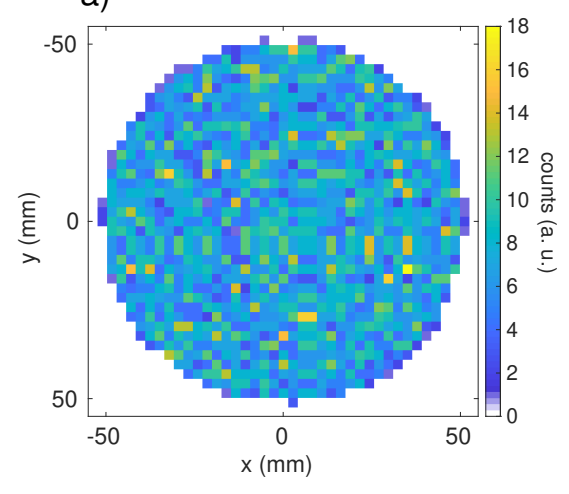

b)

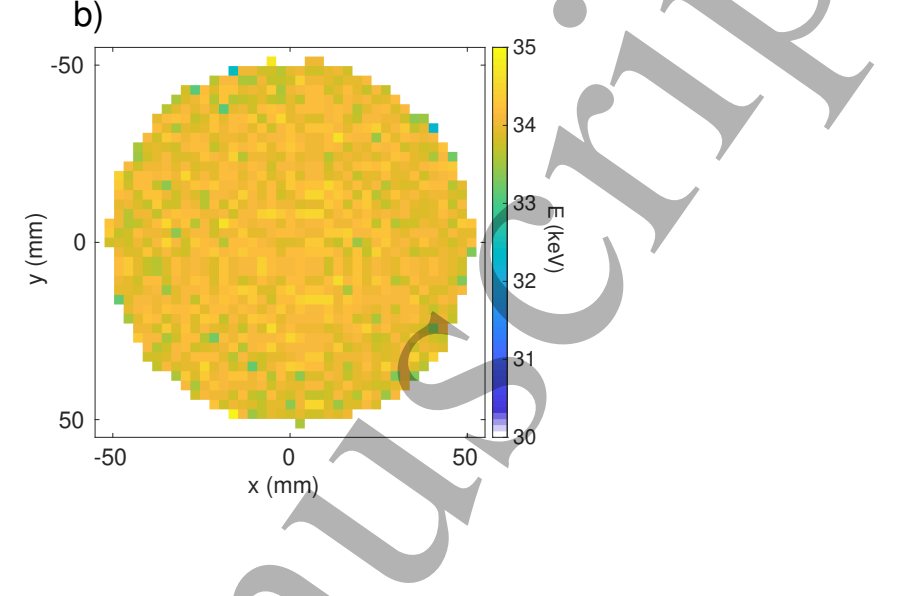

Figure 5. Radiation field of the simulated HE beam at a distance of $50 \mathrm{~m}$ from the IP. a) Intensity distribution. b) Energy distribution.

stenosis has a length $l_{S}$ and the vessel diameter reduces from the nominal value $t_{C M}$ to $t_{S}$. The stenosis degree is $G=\left(t_{C M}-t_{S}\right) / t_{C M}$ (Sarnelli et al. 2006). In our analysis, vessels with variable diameter and stenosis degree were considered. Finally, a layer of thickness $t_{B}$ was included to take into account the attenuation due to the rib bone. The rib bone elemental composition and its density were defined according to ICRU report 46 (ICRU 1992). Some parameters of the phantom such as thickness, density and attenuation coefficients of the materials are listed in table 1 , where the superscripts \pm indicate the two energies $E^{-}=31.7 \mathrm{keV}$ and $E^{+}=34.0 \mathrm{keV}$, respectively. At these energies, modern solid state detectors feature a detection efficiency close to $100 \%$, for this reason we set $\varepsilon=1$ in our model. The pixel area of the detector was supposed to be 
$F=0.35 \mathrm{~mm} \times 0.35 \mathrm{~mm}$, since this size is compatible with that of the detector pixel used for angiography at synchrotron beamlines dedicated to medical imaging, such as ID17 at ESRF (Elleaume et al. 1999) and HASYLAB at DESY (Dill et al. 1998). From equation (17), it follows that the fluence required to obtain a given $S N R$ depends inversely on $\varepsilon$ and $F$. Therefore, if a lower detection efficiency or a smaller pixel are considered, the required fluence gets higher and its relative increases is equal to the relative decrease of $\varepsilon$ and $F$.

Table 1. Phantom parameters used in the analytical model

\begin{tabular}{lllll}
\hline & thickness $(\mathrm{cm})$ & $\mu^{-} / \rho\left(\mathrm{cm}^{2} / \mathrm{g}\right)$ & $\mu^{+} / \rho\left(\mathrm{cm}^{2} / \mathrm{g}\right)$ & $\rho\left(\mathrm{g} / \mathrm{cm}^{3}\right)$ \\
\hline Iodine & - & 7.1100 & 33.5200 & 4.93 \\
Bone & 1.0 & 0.8062 & 0.6888 & 1.41 \\
Soft tissue & 19.0 & 0.3501 & 0.3200 & 1.06 \\
\hline
\end{tabular}

In a first study, the photon fluence required to obtain a signal-to-noise ratio of 5 for a region perfused with a variable concentration of contrast/medium $c_{C M}$ was evaluated following the approach described in section 2.3. As it is well known, a value of $S N R=5$ is required to fully distinguish a detail over a background area, according to the Rose model (Rose 1973). Indeed, in this model the area of the object to be imaged is taken as the sampling area for noise so as to determine the threshold $S N R$ (Wagner 1977). If the system is quantum limited, such detectability threshold will allow one to estimate the minimum exposure level that is required for the detection of image signals associated with a specific radiological examination (Taibi 2004). For a review on the signal detection theory and the limitations of the Rose model, the reader is referred to the excellent work of Burgess (Burgess 1999). According to Burgess, the Rose model is a good approximation of the ideal observer under a limited scope of ideal conditions, i.e. precisely known feature at precisely known position. This means that we can only here estimate the fluence rate for the specific imaging task but the actual visual detection of an iodinated region having area $A$ in a clinical context would require a model observer study (see for instance the paper of (Sisini et al. 2012) and references therein). Yet, in our previous study on the physical image quality of KES (see (Sarnelli et al. 2004)) we performed a comparative study between experimental data and theoretical expectations, and showed that there is a very good agreement between SNR data measured on vessels filled with iodine and the SNR data calculated via the Rose model.

The considered region had a thickness $t_{C M}=2 \mathrm{~mm}$ and a transverse (with respect to the impinging X-rays) area equal to $t_{C M} \times l_{C M}$. The detail length $l_{C M}$ was set to 2 $\mathrm{mm}$ or $5 \mathrm{~mm}$ to make a direct comparison with the case of a stenotic vessel. A square cross-section with side equal to the vessel diameter is assumed for the evaluation of the signal. This approximation is based on the work of (Wagner 1977), where the sampling aperture of some details of clinical interests were calculated. In the case of a blood vessel, the one-dimensional sampling aperture is 0.93 times the vessel diameter, thus conferming the validity of our approximation. The iodine concentration was considered to vary between 1 and $20 \mathrm{mg} / \mathrm{ml}$, since this is the typical range of concentrations detected during clinical trials carried out at synchrotron facilities, due to the dilution of the iodinated solution in the blood circulation after the venous

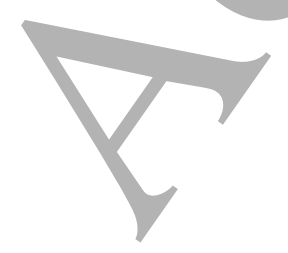


injection (Hughes et al. 1983). The same type of study was also carried out for a fixed iodine concentration $c_{C M}=10 \mathrm{mg} / \mathrm{ml}$ and varying the vessel diameter between $0.2 \mathrm{~mm}$ and $6 \mathrm{~mm}$. The parameters used in the calculations were set according to typical values reported in the literature (Bertrand et al. 2005, Dix et al. 1996, Sarnelli et al. 2005).

The stenosis detectability was also investigated. The signal due to a stenotic vessel is the difference between the signal of the non-stenotic and stenotic portion (Sarnelli et al. 2006). If the transversal area of the stenosis is $A=t_{S} \cdot l_{S}$, using the definition of stenosis degree $G$ given above, the signal can be expressed as

$$
S=\left(t_{C M}-t_{S}\right) \cdot c_{C M}=t_{C M} \cdot G \cdot c_{C M} .
$$

Starting from a non-stenotic diameter of $2 \mathrm{~mm}$, the photon fluence $\phi_{0}$ required to satisfy the Rose criterion was calculated as a function of the stenosis degree for various iodine concentrations and stenosis lengths. In this case, the dependence of $\phi_{0}$ on $c_{C M}$, $l_{S}$ and $G$ can be made more apparent substituting equation (19) in equation (17) and noting that the number of detector pixel corresponding to the stenosis segment is $n=t_{S} \cdot l_{S} / F$, where $t_{S}=t_{C M}(1-G)$, thus obtaining

$$
\phi_{0} \propto \frac{1}{t_{C M}^{3} c_{C M}^{2} l_{S} G^{2}(1-G)} .
$$

As a further analysis, the photon fluence required to obtain $S N R=5$ was also calculated, for a non-stenotic vessel of $2 \mathrm{~mm}$ and various contrast medium concentrations, as a function of the energy separation between two monoenergetic beams bracketing the iodine K-edge. Moreover, the ratio of the residual bone signal with respect to that of iodine was evaluated for different values of iodine mass thickness. According to (Rutt et al. 1983), this ratio is

$$
R_{B, I}=\frac{\left[\left(\mu_{T}^{+} / \mu_{T}^{-}\right) \mu_{B}^{-}-\mu_{B}^{+}\right]}{\left[\left(\mu_{T}^{+} / \mu_{T}^{-}\right) \mu_{I}^{-}-\mu_{I}^{+}\right]} \frac{t_{B} \cdot \rho_{I}}{t_{C M} \cdot c_{C M}},
$$

where, as already mentioned, the subscripts $T, B, I$ and $C M$ indicate soft tissue, bone, iodine and the contrast medium solution, respectively, $\rho_{I}$ is the iodine density and $\mu^{ \pm}$indicates the attenuation coefficient of the materials at the energy of the two monochromatic beams. It is worth noting that the expression $(\rho t)_{I} \simeq t_{C M} \cdot c_{C M}$, which comes from equations (6) and (13), is used for the iodine mass thickness.

\section{Results and discussion \\ 4.1. Vessel detectability}

The photon fluence $\phi_{0}$ required to achieve $S N R=5$ for the non-stenotic portion of a vessel with diameter (thickness) $t_{C M}=2 \mathrm{~mm}$ is reported in figure 7 as a function of the iodine concentration. As it can be seen, the higher $c_{C M}$ the lower $\phi_{0}$ and a fluence less than $10^{8} \mathrm{ph} \mathrm{mm^{-2 }}$ is required to satisfy the Rose criterion for $c_{C M}>3.1$ $\mathrm{mg} / \mathrm{ml}$, while a fluence less than $10^{7} \mathrm{ph} \mathrm{mm}^{-2}$ is sufficient if $c_{C M}>10 \mathrm{mg} / \mathrm{ml}$.

On the other hand, figure 8 shows the fluence required to satisfy the Rose criterion for a fixed iodine concentration and variable vessel diameter. As it can be seen, $10^{8}$ ph $\mathrm{mm}^{-2}$ are sufficient to image a vessel with diameter larger than $1 \mathrm{~mm}$. Moreover, the longer the portion of vessel to image the smaller the required fluence. 
Inverse Compton sources: a new opportunity for K-edge subtraction imaging

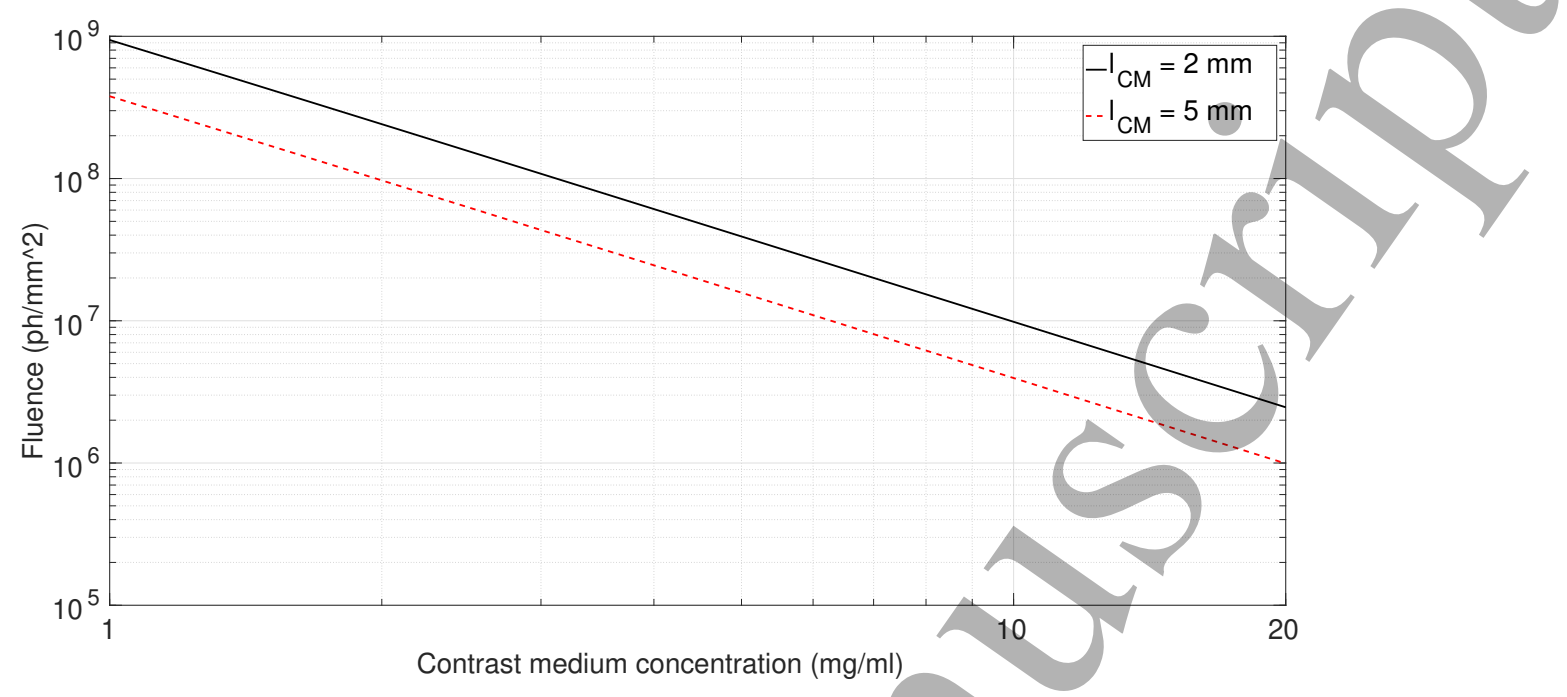

Figure 7. Photon fluence required to obtain a $S N R$ of 5 as a function of the iodinated contrast medium concentration for a vessel with $2 \mathrm{~mm}$ diameter. Two values of detail length $l_{C M}$ were considered.

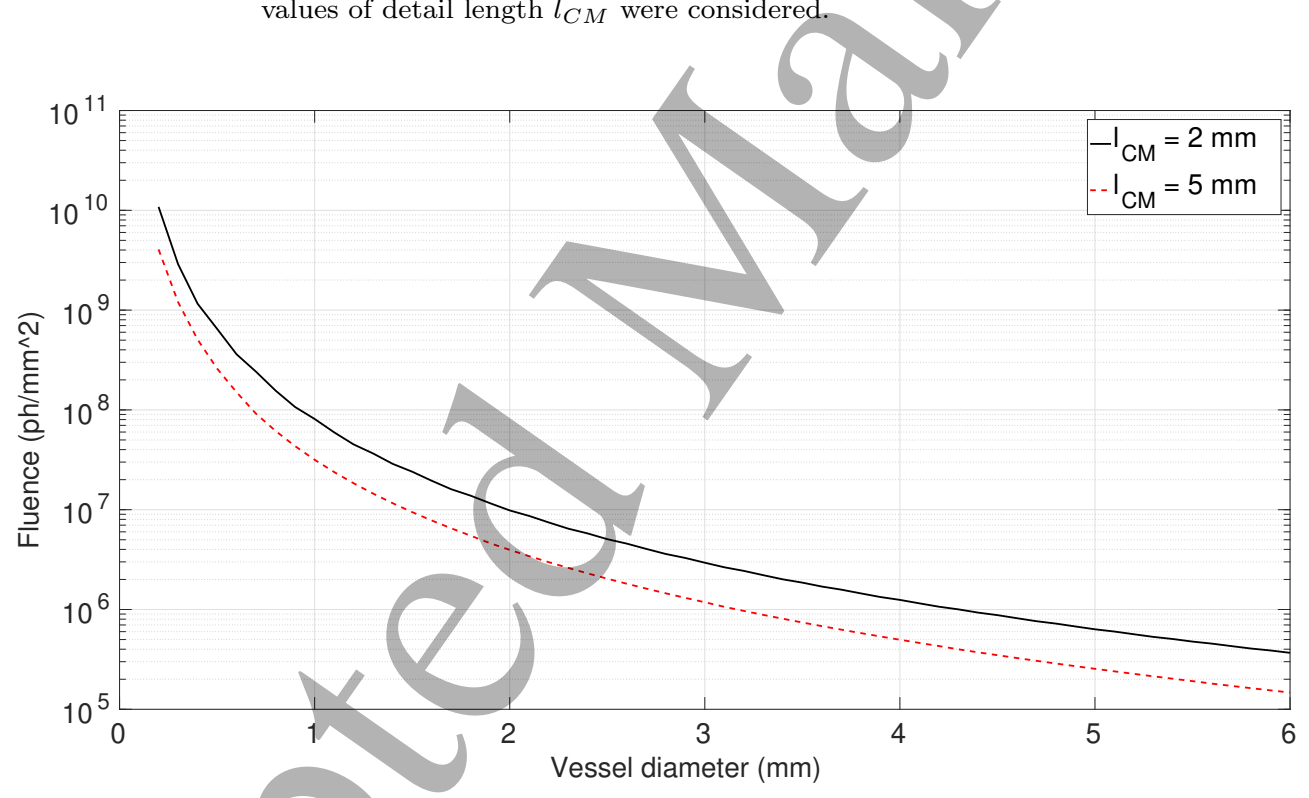

Figure 8. Photon fluence required to obtain a $S N R$ of 5 as a function of the vessel diameter for an iodine concentration of $10 \mathrm{mg} / \mathrm{ml}$. Two values of detail length $l_{C M}$ were considered.

\subsection{Detectability of stenoses}

Figure 9 shows the fluence required to image a stenosis ranging from $10 \%$ to $90 \%$ of the nominal vessel diameter $t_{C M}=2 \mathrm{~mm}$. Two values of the stenosis length $l_{S}$ and iodine concentration $c_{C M}$ were taken into account. It can be seen that the fluence decreases when these parameters increase because they cause an increment of the detection area

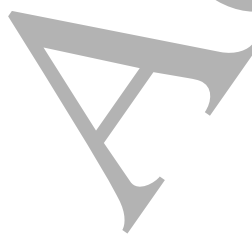


and the signal, respectively. This behaviour is summarised in equation (20), which also explains the minimum of $\phi_{0}$ in correspondence of a stenosis degree of about $67 \%$. It is worth noting that a fluence of about $10^{8} \mathrm{ph} \mathrm{mm}^{-2}$ is, in general, sufficient to image a severe stenosis $(G>50 \%)$.

\subsection{Peak energy separation}

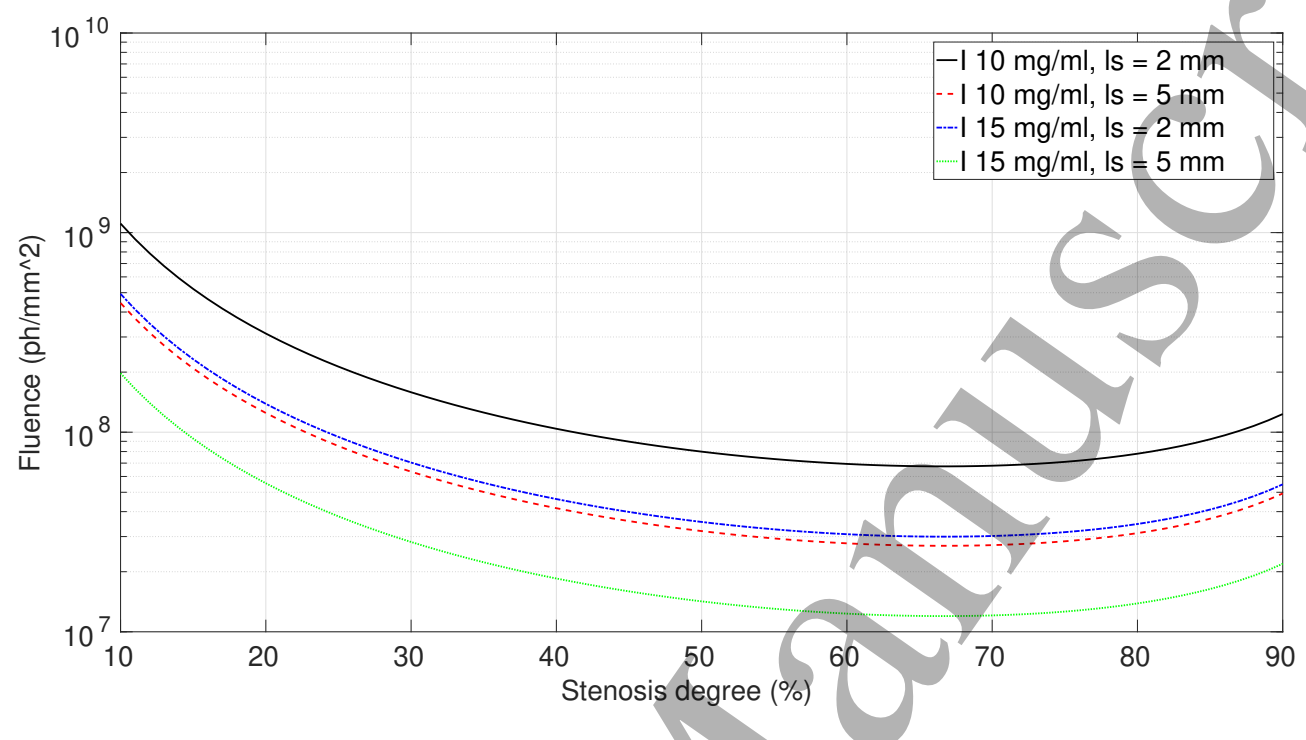

Figure 9. Photon fluence required to obtain a $S N R$ of 5 as a function of the stenosis degree, for two values of contrast medium concentration and stenosis length and a nominal vessel diameter $t_{C M}=2 \mathrm{~mm}$.

In the previous analysis, the energy of the two x-ray beams was fixed. However, for the general case of a vessel without stenosis, we considered also the case of two monochromatic beams with energy separation $\Delta E$ symmetrically variable with respect to the iodine K-edge. Figure 10 shows the photon fluence $\phi_{0}$ required to satisfy the Rose criterion as a function of $\Delta E$, for a vessel with a diameter of $2 \mathrm{~mm}$ and various iodine concentrations. As expected, $\phi_{0}$ decreases if $c_{C M}$ increases, while an increase in $\Delta E$ causes an increase in $\phi_{0}$. Indeed, the signal is proportional to $c_{C M}$ but is practically independent from the energy of the beams. On the contrary, due to the monotonic behaviour of the attenuation coefficient of the background materials, $\sigma_{P}$ decreases if the two peaks are closer in energy.

An important aspect to consider is the bone residual signal, due to an imperfect cancellation when the dual-energy algorithm described before is applied. To this purpose, the ratio of the bone signal to the iodine signal $R_{B, I}$ was calculated as a function of $\Delta E$, for the phantom depicted in figure 6 . A rib bone with a thickness of 1 $\mathrm{cm}$ (mass thickness $(\rho t)_{B}=1.41 \mathrm{~g} / \mathrm{cm}^{2}$ ) and three different values of mass thickness for iodine $(\rho t)_{I}$ were considered. Therefore, the ratio $(\rho t)_{B} /(\rho t)_{I}$ ranged from 467 to 705. The result of this analysis is shown in figure 11. As expected, the higher the ratio $(\rho t)_{B} /(\rho t)_{I}$, the higher the residual bone signal. For $(\rho t)_{I}=3 \mathrm{mg} / \mathrm{cm}^{2}$, which corresponds, for example, to a vessel with a diameter of $2 \mathrm{~mm}$ filled with $15 \mathrm{mg} / \mathrm{ml}$ of iodine, the bone signal is lower than the iodine signal, provided that the peak energy

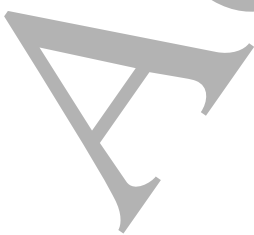




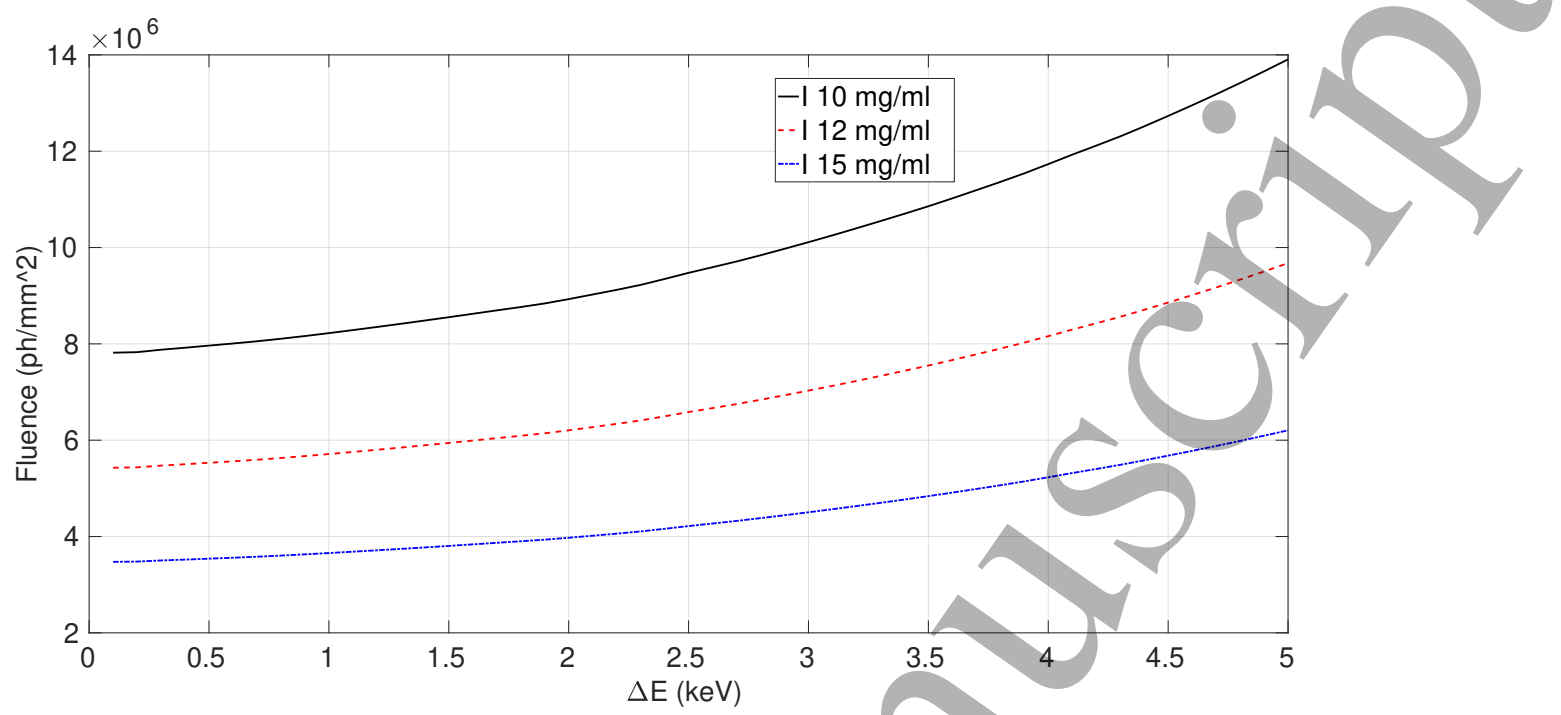

Figure 10. Photon fluence required to obtain a $S N R$ of 5 as a function of the energy separation between the two monochromatic beams, for $t_{C M}=2 \mathrm{~mm}$ and various iodine concentrations.

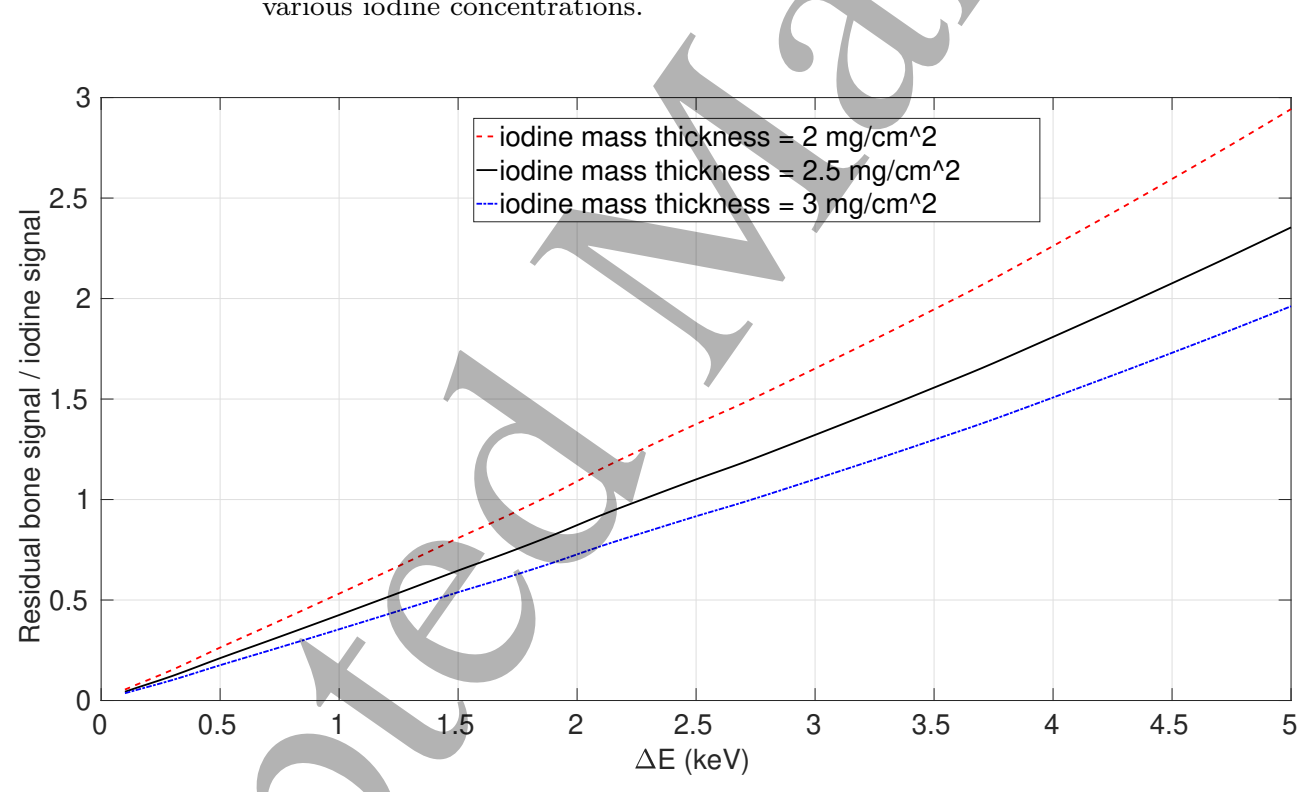

Figure 11. Ratio of the residual bone signal with respect to that of iodine as a function of the energy separation between the two monochromatic beams. Different mass thicknesses were considered for iodine, while $(\rho t)_{B}=1.41 \mathrm{~g} / \mathrm{cm}^{2}$.

separation is less than $2.7 \mathrm{keV}$. For the energies $E^{-}=31.7 \mathrm{keV}$ and $E^{+}=34.0 \mathrm{keV}$, bone produces a signal that is $84 \%$ of the iodine signal. At these energies, the signal of bone is equal to the signal produced by $2.5 \mathrm{mg} / \mathrm{cm}^{2}$ of iodine. It is worth noting that these evaluations were carried out under the assumption of perfect monochromaticity for the two X-ray beams. As the relative energy separation decreases and becomes 
comparable with the energy bandwidth, a more accurate analysis, taking into account the overlap of the two X-ray spectra, would be desirable.

\subsection{Performance of inverse Compton sources}

As described in section 2.1, inverse Compton sources are characterised by a strong correlation between energy and angle of the scattered photons. This feature permits to control the energy bandwidth of the emitted radiation through collimation. The collimation angle $\theta_{\max }$, and therefore the maximum divergence of the emitted photons, has to be set in such a way to limit as much as possible the overlap of the energy spectra of the two quasi-monochromatic X-ray beams required for KES imaging. Furthermore, the uniformity of the radiation field at a given distance from the IP can be enhanced if $\theta_{\max }$ is set close to the critical value. The drawback of reducing $\theta_{\text {max }}$ is, of course, the reduction of the number of photons within the acceptance angle $N_{\psi}$. For this reason, $\mathrm{X}$-ray beams with a high brilliance $\left(\mathrm{ph} \mathrm{s}^{-1} \mathrm{BW} \%^{-1} \mathrm{~mm}^{-2} \mathrm{mrad}^{-2}\right)$ are desired. The electron bunch charge, the laser pulse energy and collision frequency have to be as high as possible to obtain high luminosities and therefore a large number of emitted photons. Furthermore, the energy spread and normalised emittance of the electron beam are key parameters to control the brilliance of the emitted X-ray beams. They have to be as small as possible in order to obtain brilliant beams. Once the collimation angle is set, the radiation field diameter $D$ can be estimated using equation (3), which states that $D$ increases linearly with $d$ and $\theta_{\max }$. On the other hand, the photon flux at a distance $d$ from the IP increases with $\theta_{\max }^{2}$, but decreases with the square of $d$. Therefore, for an ICS, a trade-off exists between photon flux on the sample and size of the radiation field.

From the previous subsections, it turns out that a photon fluence of the order of $10^{8} \mathrm{ph} \mathrm{mm}^{-2}$ is required to obtain images of most coronary arteries with an adequate $S N R$ through the KES method. This value is consistent with those reported in the literature describing clinical studies carried out at synchrotron beamlines. Indeed, the paper of (Dix et al. 1996) reports a number of $3 \times 10^{11} \mathrm{ph} \mathrm{mm}^{-2} \mathrm{~s}^{-1}$ to obtain clinical images of the coronary arteries at HASYLAB. A very similar value, i.e. $6 \times 10^{11} \mathrm{ph}$ $\mathrm{mm}^{-2} \mathrm{~s}^{-1}$, is shown by (Elleaume et al. 1999) in the ESRF system specifications. Since in both studies the exposure time per line of the detector was $1 \mathrm{~ms}$, the integrated photon fluence is comparable to the value reported above. In those studies, the beam intensity was mainly limited by the maximum dose deliverable to skin of the patients (few hundreds of miSv for a complete examination). For this reason, values of fluence much higher than $10^{8} \mathrm{ph} \mathrm{mm}^{-2}$ are not even desirable. Using equation (18), it is possible to calculate that such value of photon fluence corresponds to a skin dose of about $26 \mathrm{mSv}$ to acquire a couple of images. As a comparison, the value of skin-dose reported for the ESRF angiography system is $32 \mathrm{mSv}$ per image couple (Elleaume et al. 1999). Finally, an important requirement is the radiation field diameter, which has to be at least $10 \mathrm{~cm}$.

Most of the ICSs currently available worldwide provide X-ray beams that achieve this radiation field size with a flux three or four orders of magnitude smaller than the required one. However, novel sources are much more intense. For example, MuCLS (Eggl et al. 2016) can provide quasi-monochromatic beams (5\% bandwidth) with an energy of up to $35 \mathrm{keV}$, delivering about $10^{10} \mathrm{ph} / \mathrm{s}$ in a cone with a semi-aperture of $2 \mathrm{mrad}$, which means a flux of about $10^{6} \mathrm{ph} \mathrm{mm}^{-2} \mathrm{~s}^{-1}$ for a beam size of $10 \mathrm{~cm}$. Moreover, ThomX is expected to be able to produce X-ray beams with energy up to 
$90 \mathrm{keV}$ and bandwidth of $1-10 \%$ that encompass from $10^{11}$ to $10^{13} \mathrm{ph} / \mathrm{s}$ in a cone with a semi-aperture of $2 \mathrm{mrad}$ (Variola et al. 2014), leading to a flux ranging from $10^{7}$ to $10^{9} \mathrm{ph} \mathrm{mm}^{-2} \mathrm{~s}^{-1}$ for a beam size of $10 \mathrm{~cm}$. MariX aims to deliver X-ray beams with intensities comparable to those of ThomX, featuring the additional capability of changing the beam energy in a time of the order of few hundreds of ms (Serafini et al. 2018, Drebot et al. 2018a). These considerations justify the interest in further analysing the performance of KES angiography using an ICS.

Due the large number of parameters involved, the quality of the X-ray beam emitted from an ICS can be extremely different, depending on the features of the colliding beams, which in their turn depend strongly on the complexity and the cost of the ICS itself. In order to perform a quantitative analysis, the collision parameters reported in table 2 were set. They were chosen according to the characteristics of a medium size facility based on inverse Compton, such as MariX. The mean peak energy was set to $31.7 \mathrm{keV}$ and $34.0 \mathrm{keV}$ for the LE and $\mathrm{HE}$ beam respectively, to be consistent with previous treatment. The features of the resulting X-ray beams were analytically calculated using the scaling laws reported in the literature (Curatolo et al. 2017). A collimation angle of $2.23 \mathrm{mrad}$ was set to achieve a relative energy bandwidth of $1.5 \%$ and guarantee a complete separation between the two peaks. The number of collimated photons emitted per second is $N_{\psi}=1.25 \times 10^{14}$. A radiation field with a diameter of $15 \mathrm{~cm}$, which is a size that allows to fully image the heart, is obtained at a distance of $33.6 \mathrm{~m}$ from the IP. As shown in figure 12, the photon flux is about $3.5 \times 10^{8} \mathrm{ph} \mathrm{mm}^{-2} \mathrm{~s}^{-1}$ at this distance.

Table 2. Colliding beams used to evaluate the potential of ICSs

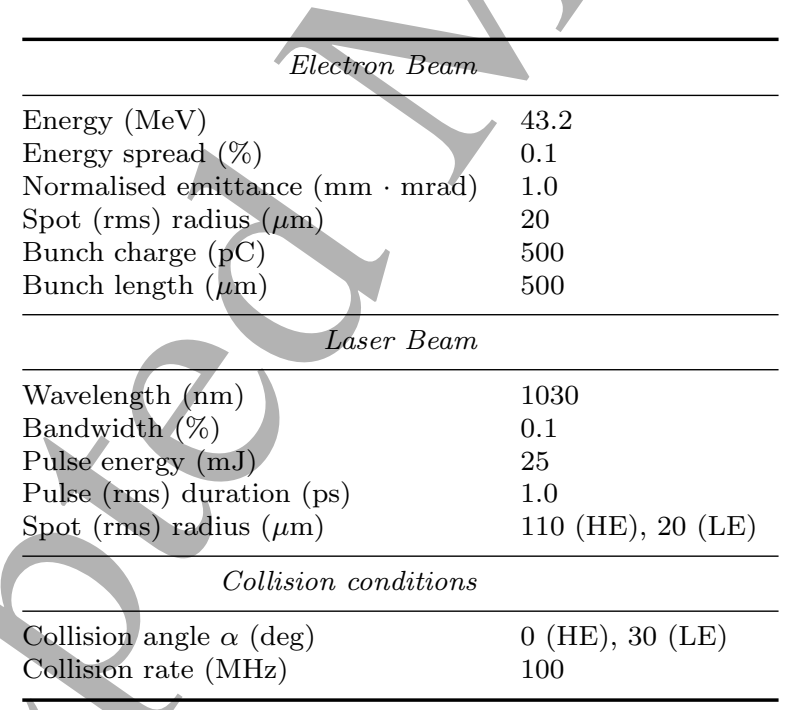

More in detail, $d$ should be chosen as a function of the fluence required and the maximum time allowed to perform a KES procedure. Concerning the latter point, it is known that the heart stands still for about $100 \mathrm{~ms}$ between a beat and the following (Gary et al. 1993) and therefore, the exposure time for each image should not exceed this value to avoid motion artifacts. The effective exposure time can be increased provided that the acquisition is synchronised with the heart rate through

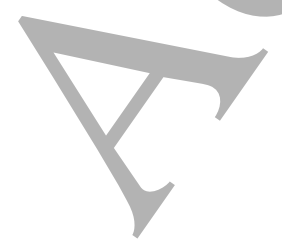




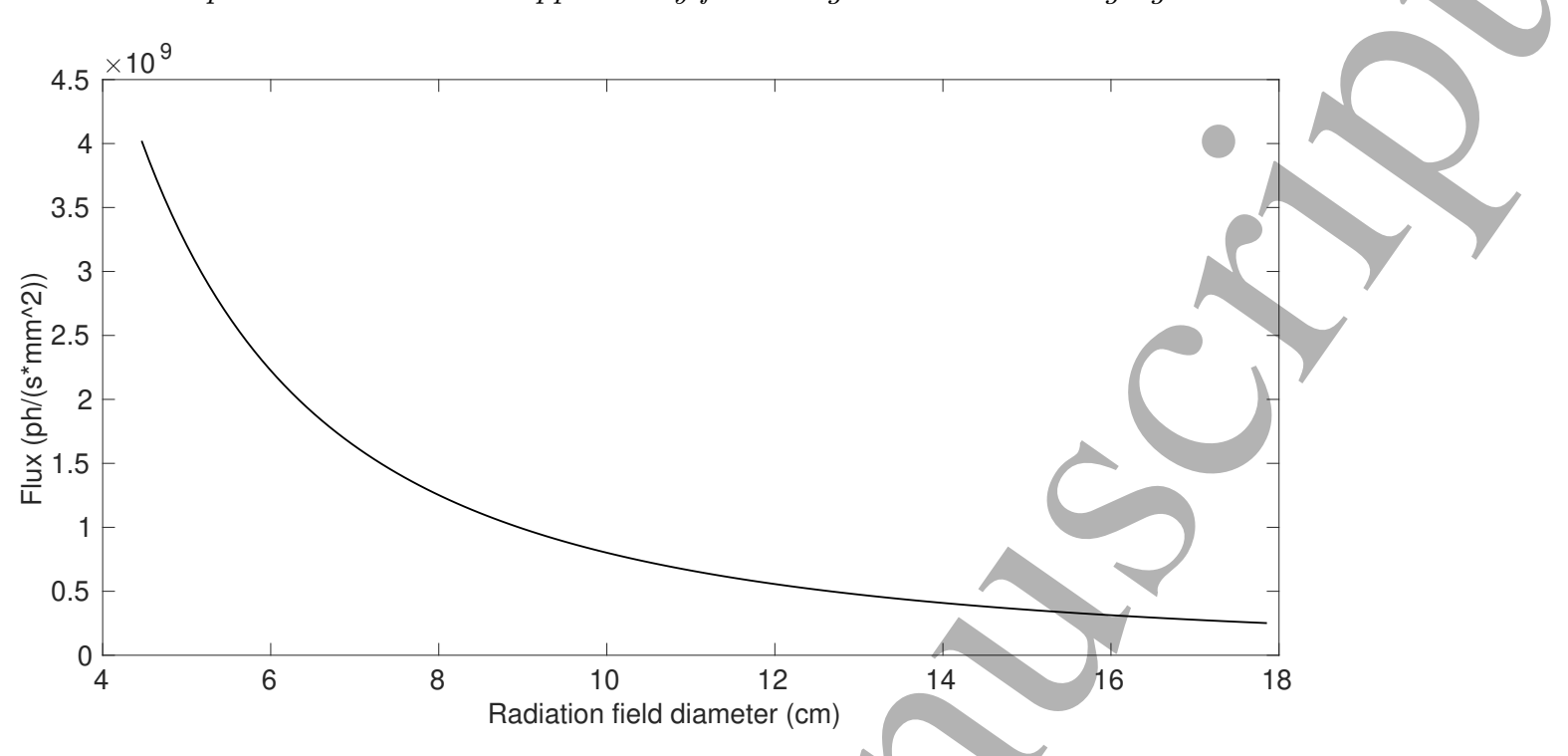

Figure 12. Flux versus diameter of the radiation field for the beams described in table 2 .

electrocardiography (Kroft et al. 2007). Indeed, in this case, it is possible to sum the signals related to few consecutive heart beats. If 3 beats are exploited, the effective exposure time for each beam is $300 \mathrm{~ms}$. Therefore, the fluence of the X-rays emitted by an ICS based on colliding beams with the features reported in table 2 is about $10^{8}$ $\mathrm{ph} \mathrm{mm}^{-2}$ for a radiation field with a diameter of $15 \mathrm{~cm}$. As mentioned above, such a value of $\phi_{0}$ is sufficient in most of the cases. Indeed, it allows to image a vessel of $2 \mathrm{~mm}$ filled with less than $3.5 \mathrm{mg} / \mathrm{ml}$ of iodine and distinguish a $2 \mathrm{~mm}$ long stenosis of $40 \%$, with $10 \mathrm{mg} / \mathrm{ml}$ of iodine. By considering that the patient has to be exposed to the HE and LE beam separately for the time necessary to acquire the signal due to three heart beats and that a certain amount of time is necessary for changing the beam energy, the total time required for a complete acquisition is estimated to be of the order of $6 \mathrm{~s}$, for the simulated ICS. Since during the acquisition the heart rate has to be stable and the patients has to breath-hold, this time can not be increased arbitrarily. Hence, if smaller details have to be imaged with a lower contrast medium concentration or if a less-intense ICS is used, the required fluence can be obtained by reducing $d$, although the radiation field gets smaller. The radiation field size can be maintained by increasing the angular acceptance and thus, the photon flux. However, in this case, the bandwidth of the two quasi-monochromatic X-ray beams increases and they could overlap. The angular acceptance can be also increased in order to have a shorter propagation distance $d$, hence a more compact source. The actual possibility of increasing the angular acceptance is the result of a trade-off that has to be evaluated depending on the specific ICS and application.

Finally, if a smaller residual bone signal is required, the energy separation $\Delta E$ between the two nearly monochromatic peaks bracketing the iodine K-edge has to be reduced. However, due to the low energy tail in the collimated beam energy spectra (see figure 4), a significant fraction of the HE peak is below the contrast medium Kedge and overlaps with the LE peak as $\Delta E$ gets smaller and smaller. This effect causes

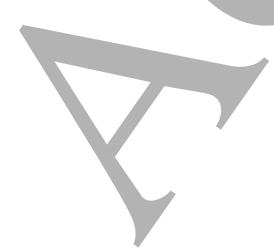


artifacts in the iodine image if the simple dual-energy algorithm is used (Thomlinson et al. 2018). Nevertheless, this case can be treated by applying a polychromaticity correction algorithm (Contillo et al. 2015). Alternatively, possible artifacts due to highly absorbing structures can be limited by applying the method described in (Kulpe et al. 2018), without reducing the energy separation $\Delta E$.

\section{Conclusions}

This work aims to give a first quantitative approach to the K-edge subtraction technique by means of an inverse Compton source. A study concerning the image quality obtainable in the case of coronary angiography was carried out by evaluating the detail detectability of a specific phantom. In agreement with clinical tests carried out at synchrotron beamlines, the study demonstrates that a photon fluence of the order of $10^{8} \mathrm{ph} \mathrm{mm^{-2 }}$ is sufficient to image most of coronary arteries and their possible stenoses with an adequate $S N R$. The most recent ICSs, currently under commissioning or in proposal stage, have the potential to deliver intense quasimonochromatic beams capable of achieving the required fluence with an acquisition time of few hundreds of ms. The moderate divergence (few mrad) of these beams permits to achieve useful radiation field sizes at distances from the IP well below 100 $\mathrm{m}$ and full-field dual-energy images could be obtained without the need of scanning the beam. If required, the collimation angle and the distance from the interaction point can be set to enhance the photon flux at the expenses of the beam monochromaticity and the radiation field size. A useful characteristics of ICSs is the ability of delivering high photon fluxes also at energies higher than iodine K-edge. This could open the way to the investigation of KES imaging with contrast agents having higher K-edge, such as Gadolinium, whose K-edge is at $50.2 \mathrm{keV}$. Another important characteristics of ICSs is the compactness of the beamline and accelerator with respect to synchrotrons for the production of monochromatic X-rays. This féature together with those previously discussed, makes ICSs very promising X-ray sources for KES imaging research and potential clinical applications.

\section{References}

Alvarez R E \& Macovski A 1976 Energy-selective reconstructions in x-ray computerised tomography Physics in Medicine \&5 Biology 21(5), 733.

Bacci A, Puppin E, Agostino R, Alesini D, Gatti G, Petrillo V, Borgese G, Anania M P, Palmer D, Fabris A et al. 2014 The STAR project 5th International Particle Accelerator Conference (IPAC 2014).

Bertrand B, Esteve F, Elleaume H, Nemoz C, Fiedler S, Bravin A, Berruyer G, Brochard T, Renier M, Machecourt J et al. 2005 Comparison of synchrotron radiation angiography with conventional angiography for the diagnosis of in-stent restenosis after percutaneous transluminal coronary angioplasty European Heart Journal 26(13), 1284-1291.

Brown W J \& Hartemann F V 2004 Three-dimensional time and frequency-domain theory of femtosecond x-ray pulse generation through Thomson scattering Physical Review Special Topics-Accelerators and Beams 7(6), 060703.

Burgess A E 1999 The Rose model, revisited JOSA A 16(3), 633-646.

Carroll F 2003 Tunable, monochromatic x-rays: An enabling technology for molecular/cellular imaging and therapy Journal of Cellular Biochemistry 90(3), 502-508.

Chen P, Horton-Smith G, Ohgaki T, Weidemann A \& Yokoya K 1995 CAIN: Conglomérat d'ABEL et d'Interactions Non-linéaires Nuclear Instruments and Methods in Physics Research Section A: Accelerators, Spectrometers, Detectors and Associated Equipment 355(1), 107 - 110. Gamma-Gamma Colliders. 
Contillo A, Di Domenico G, Cardarelli P, Gambaccini M \& Taibi A 2015 A novel approach to background subtraction in contrast-enhanced dual-energy digital mammography with commercially available mammography devices: Polychromaticity correction Medical Physics 42(11), 6641-6650.

Curatolo C, Drebot I, Petrillo V \& Serafini L 2017 Analytical description of photon beam phase spaces in inverse Compton scattering sources Physical Review Accelerators and Beams 20(8), 080701.

Dabin Y, Draperi A, Elleaume H, Charvet A, Brochard T, Perez M, Nemoz C, Blattmann G, Renier $\mathrm{M}$, Fournier $\mathrm{F}$ et al. $2001 \mathrm{~A}$ patient positioning system for the ESRF medical imaging facility Nuclear Instruments and Methods in Physics Research Section A: Accelerators, Spectrometers, Detectors and Associated Equipment 467, 1342-1345.

Dill T, Dix W, Hamm C, Jung M, Kupper W, Lohmann M, Reime B \& Ventura R 1998 Intravenous coronary angiography with synchrotron radiation European Journal of Physics 19(6), 499.

Dix W, Besch H, Graeff W, Hamm C, Illing G, Kupper W, Lohmann M, Meinertz T, Menk R, Reime B et al. 1996 Intravenous coronary angiography with synchrotron radiation Physica Scripta 1996(T61), 51.

Dix W R, Kupper W, Dill T, Hamm C, Job H, Lohmann M, Reime B \& Ventura R 2003 Comparison of intravenous coronary angiography using synchrotron radiation with selective coronary angiography Journal of Synchrotron Radiation 10(3), 219-227.

Drebot I, Petrillo V \& Serafini L 2017 Two-colour X-gamma ray inverse Compton back-scattering source EPL (EuroPhysics Letters) 120(1), 14002.

Drebot I et al. 2018a Multi colour x-gamma ray inverse Compton back-scattering source in 'Proc. 9th Int. Particle Accelerator Conf. (IPAC18)' JACOW Publishing, Geneva, Switzerland.

Drebot I et al. 2018b Optimisation Study of the Fabry-Pérot Optical Cavity for the MARIX/BRIXS Compton X-Ray Source in 'Proc. 9th Int. Particle Accelerator Conf. (IPAC18)' JACOW Publishing, Geneva, Switzerland.

Eggl E, Dierolf M, Achterhold K, Jud C, Günther B, Braig E, Gleich B \& Pfeiffer F 2016 The Munich compact light source: initial performance measures Journal of Synchrotron Radiation 23(5), 1137-1142.

Eggl E, Mechlem K, Braig E, Kulpe S, Dierolf M, Günther B, Achterhold K, Herzen J, Gleich B, Rummeny E et al. 2017 Mono-energy coronary angiography with a compact synchrotron source Scientific Reports 7, 42211.

Elleaume H, Charvet A, Berkvens P, Berruyer G, Brochard T, Dabin Y, Dominguez M, Draperi A, Fiedler S, Goujon G et al. 1999 Instrumentation of the ESRF medical imaging facility Nuclear Instruments and Methods in Physics Research Section A: Accelerators, Spectrometers, Detectors and Associated Equipment 428(2-3), 513-527.

Elleaume H, Fiedler S, Esteve F, Bertrand B, Charvet A, Berkvens P, Berruyer G, Brochard T, Le Duc G, Nemoz C et al. 2000 First human transvenous coronary angiography at the European Synchrotron Radiation Facility Physics in Medicine and Biology 45(9), L39.

Esarey E, Ride S K \& Sprangle P 1993 Nonlinear Thomson scattering of intense laser pulses from beams and plasmas Physical Review E 48(4), 3003.

Fredenberg E 2018 Spectral and dual-energy x-ray imaging for medical applications Nuclear Instruments and Methods in Physics Research Section A: Accelerators, Spectrometers, Detectors and Associated Equipment 878, 74 - 87. Radiation Imaging Techniques and Applications.

Gary C, Piestrup M, Boyers D, Pincus C, Pantell R \& Rothbart G 1993 Noninvasive digital energy subtraction angiography with a channeling-radiation x-ray source Medical Physics 20(5), 1527-1535.

Golosio B, Endrizzi M, Oliva P, Delogu P, Carpinelli M, Pogorelsky I \& Yakimenko V 2012 Measurement of an inverse Compton scattering source local spectrum using k-edge filters Applied Physics Letters 100(16), 164104.

Herr W \& Muratori B 2006 Concept of luminosity CAS-CERN Accelerator School, CERN-2006-002, pg 361-378 CERN.

Hughes E, Zeman H, Campbell L, Hofstadter R, Meyer-Berkhout U, Otis J, Rolfe J, Stone J, Wilson S, Rubenstein E et al. 1983 The application of synchrotron radiation to non-invasive angiography Nuclear Instruments and Methods in Physics Research 208(1-3), 665-675.

ICRU 1989 Tissue Substitutes in Radiation Dosimetry and Measurement. Report 44 of the International Commission on Radiation Units and Measurements (Bethesda, MD).

ICRU 1992 Photon, Electron, Proton and Neutron Interaction Data for Body Tissues. Report 46 of the International Commission on Radiation Units and Measurements (Bethesda, MD).

Jacobson B 1953 Dichromatic absorption radiography. dichromography Acta radiologica 6, 437-452.

Johnson L W, Krone R, of the Society for Cardiac Angiography R C \& Interventions 1993 Cardiac
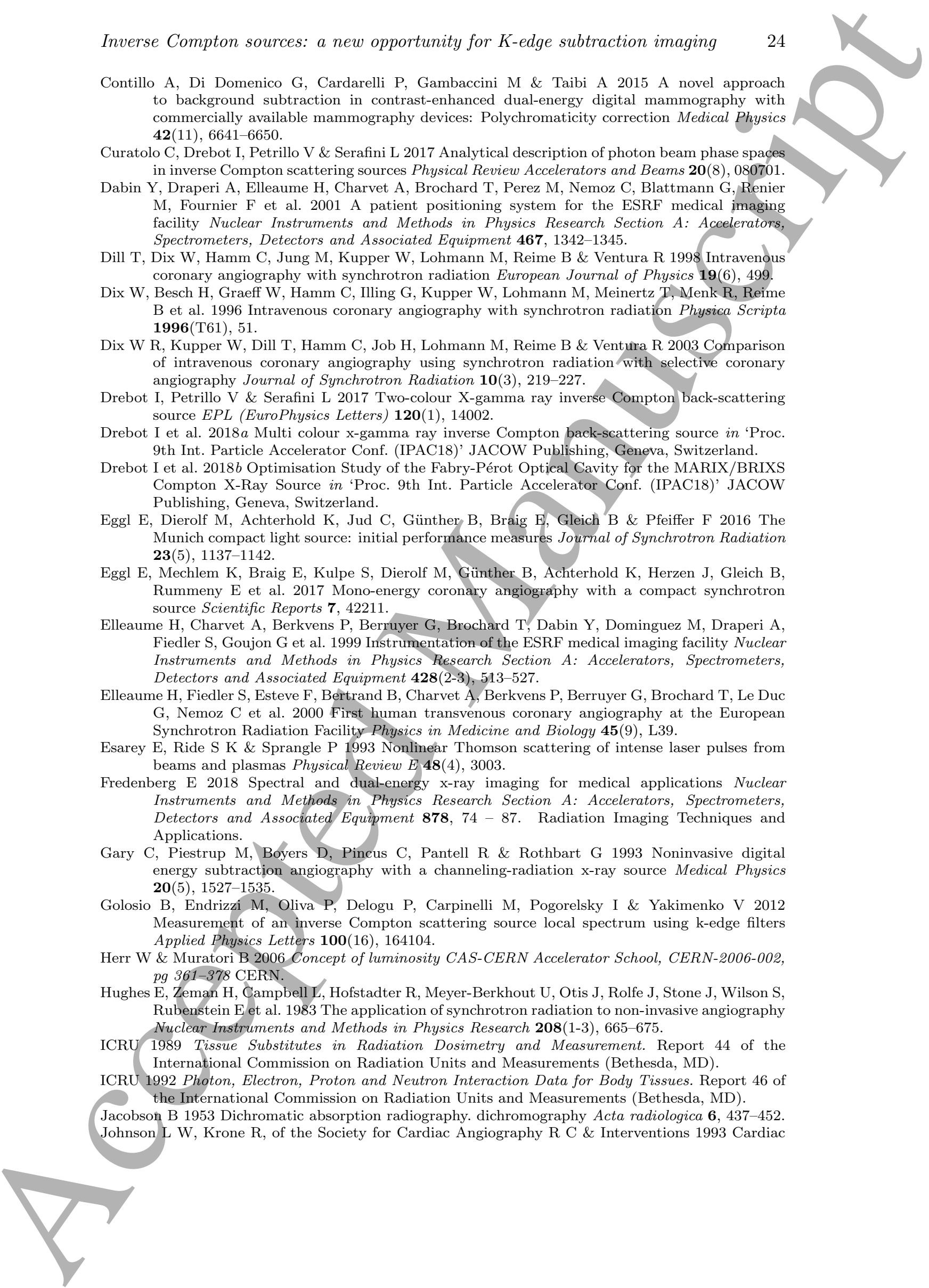
catheterization 1991: a report of the registry of the society for cardiac angiography and interventions Catheterization and cardiovascular diagnosis 28(3), 219-220.

Krafft G A \& Priebe G 2010 in 'Reviews Of Accelerator Science And Technology: Volume 3: Accelerators as Photon Sources' World Scientific pp. 147-163.

Krafft G, Johnson E, Deitrick K, Terzić B, Kelmar R, Hodges T, Melnitchouk W \& Delayen J 2016 Laser pulsing in linear Compton scattering Physical Review Accelerators and Beams 19(12), 121302.

Kroft L, De Roos A \& Geleijns J 2007 Artifacts in ECG-synchronized MDCT coronary angiography American Journal of Roentgenology 189(3), 581-591.

Kulpe S, Dierolf M, Braig E, Günther B, Achterhold K, Gleich B, Herzen J, Rummeny E, Pfeiffer F \& Pfeiffer D 2018 K-edge subtraction imaging for coronary angiography with a compact synchrotron X-ray source PLOS ONE 13(12), e0208446.

Kuroda R 2014 Inverse Compton Scattering Sources In A. Brahme (Editor in Chief.) Comprehensive BioMedical Physics, Elsevier.

Kuroda R, Toyokawa H, Yasumoto M, Ikeura-Sekiguchi H, Koike M, Yamada K, Yanagida T, Nakajyo T, Sakai F \& Mori K 2011 Quasi-monochromatic hard X-ray source via laser Compton scattering and its application Nuclear Instruments and Methods in Physics Research Section A: Accelerators, Spectrometers, Detectors and Associated Equipment 637(1), S183-S186.

Lehmann L, Alvarez R, Macovski A, Brody W, Pelc N, Riederer S J \& Hall A 1981 Generalized image combinations in dual KVP digital radiography Medical Physics 8(5), 659-667.

Maroli C, Petrillo V, Tomassini P \& Serafini L 2013 Nonlinear effects in Thomson backscattering Physical Review Special Topics-Accelerators and Beams 16(3), 030706.

Mistretta C, Ort M, Kelcz F, Cameron J, Siedband M \& Crummy A 1973 Absorption edge fluoroscopy using quasi-monoenergetic x-ray beams Investigative Radiology 8(6), 402-412.

Paternò G, Cardarelli P, Marziani M, Bagli E, Evangelisti F, Andreotti M, Gambaccini M, Petrillo V, Drebot I, Bacci A et al. 2017 A collimation system for ELI-NP Gamma Beam Systemdesign and simulation of performance Nuclear Instruments and Methods in Physics Research Section B: Beam Interactions with Materials and Atoms 402, 349-353.

Patrignani C et al. 2016 Review of particle physics Chinese Physics C 40(10), 100001.

Petrillo V, Bacci A, Zinati R B A, Chaikovska I, Curatolo C, Ferrario M, Maroli C, Ronsivalle C, Rossi A, Serafini L et al. 2012 Photon flux and spectrum of $\gamma$-rays Compton sources Nuclear Instruments and Methods in Physics Research Section A: Accelerators, Spectrometers, Detectors and Associated Equipment 693, 109-116.

Priebe G, Laundy D, Phillips P, Graham D, Jamison S, Vassilev S, Seddon E, Rosenzweig J, Krafft G, Heinzl T et al. 2010 First results from the Daresbury Compton backscattering X-ray source (COBALD) in 'Hard X-Ray, Gamma-Ray, and Neutron Detector Physics XII' Vol. 7805 International Society for Optics and Photonics p. 780513.

Ranjan N, Terzić B, Krafft G, Petrillo V, Drebot I \& Serafini L 2018 Simulation of inverse Compton scattering and its implications on the scattered linewidth Physical Review Accelerators and Beams 21(3), 030701.

Roessl E \& Proksa R 2007 K-edge imaging in x-ray computed tomography using multi-bin photon counting detectors Physics in Medicine and Biology 52(15), 4679-4696.

Rose A 1973 Vision: Human and Electronic Plenum Press: New York.

Rutt B K, Cunningham I A \& Fenster A 1983 Selective iodine imaging using lanthanum K fluorescence Medical Physics 10(6), 801-808.

Sarnelli A, Elleaume H, Taibi A, Gambaccini M \& Bravin A 2006 K-edge digital subtraction imaging with dichromatic x-ray sources: SNR and dose studies Physics in Medicine EG Biology $\mathbf{5 1}(17), 4311$.

Sarnelli A, Nemoz C, Elleaume H, Estève F, Bertrand B \& Bravin A 2005 Quantitative analysis of synchrotron radiation intravenous angiographic images Physics in Medicine EG Biology $\mathbf{5 0}(4), 725$.

Sarnelli A, Taibi A, Tuffanelli A, Baldazzi G, Bollini D, Rodriguez A C, Gombia M, Prino F, Ramello L, Tomassi E et al. 2004 K-edge digital subtraction imaging based on a dichromatic and compact X-ray source Physics in Medicine E Biology 49(14), 3291.

Serafini L, Bacci A, Bellandi A, Bertucci M, Bolognesi M, Bosotti A, Broggi F, Calandrino R, Camera F, Canella F et al. 2019 MariX, an advanced MHz-class repetition rate X-ray source for linear regime time-resolved spectroscopy and photon scattering Nuclear Instruments and Methods in Physics Research Section A: Accelerators, Spectrometers, Detectors and Associated Equipment.

Serafini L, Bacci A, Broggi F, Bosotti A, Coelli S, Curatolo C, Drebot I, Faillace L, Giannotti D, Giove D et al. 2018 The MariX source (Multidisciplinary Advanced Research Infrastructure

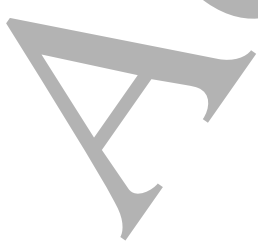


with X-rays) in 'Proc. 9th Int. Particle Accelerator Conf. (IPAC18)' JACOW Publishing, Geneva, Switzerland.

Sisini F, Zanca F, Marshall N W, Taibi A, Cardarelli P \& Bosmans H 2012 Comparison of signal to noise ratios from spatial and frequency domain formulations of nonprewhitening model observers in digital mammography Medical Physics 39(9), 5652-5663.

Sun C \& Wu Y K 2011 Theoretical and simulation studies of characteristics of a Compton light source Physical Review Special Topics-Accelerators and Beams 14(4), 044701.

Taibi A 2004 Conventional radiology In A. Del Guerra (Editor) Ionizing radiation detectors for medical imaging, World scientific.

Tang C, Huang W, Li R, Du Y, Yan L, Shi J, Du Q, Yu P, Chen H, Du T et al. 2009 Tsinghua Thomson scattering X-ray source Nuclear Instruments and Methods in Physics Research Section A: Accelerators, Spectrometers, Detectors and Associated Equipment 608(1), S70S74.

Tavakol M, Ashraf S \& Brener S J 2012 Risks and complications of coronary angiography: a comprehensive review Global Journal of Health Science 4(1), 65.

Thomlinson W, Elleaume H, Porra L \& Suortti P 2018 K-edge subtraction synchrotron x-ray imaging in bio-medical research Physica Medica 49, 58-76.

Tomassini P, Bacci A, Cary J, Ferrario M, Giulietti A, Giulietti D, Gizzi L A, Labate L, Serafini L, Petrillo V \& Vaccarezza C 2008 Linear and Nonlinear Thomson Scattering for Advanced X-ray Sources in PLASMONX IEEE Transactions on Plasma Science 36(4), 1782-1789.

Vaccarezza C, Alesini D, Anania M P, Bacci A, Biagioni A, Bisesto F, Bellaveglia M, Cardarelli P, Cardelli F, Cianchi A, Chiadroni E, Croia M, Curcio A, Delogu P, Giovenale D D, Domenico G D, Pirro G D, Drebot I, Ferrario M, Filippi F, Gallo A, Galletti M, Gambaccini M, Giribono A, Golosio B, Li W, Mostacci A, Oliva P, Palmer D, Petrillo V, Petrarca M, Pioli S, Piersanti L, Pompili R, Romeo S, Rossi A R, Scifo J, Serafini L, Suliman G \& Villa F 2016 The SPARC_LAB Thomson source Nuclear Instruments and Methods in Physics Research A $\mathbf{8 2 9}, 237-242$.

Variola A 2011 The ThomX Project in '2nd International Particle Accelerator Conference (IPAC'11)' Vol. WEOAA01 Joint Accelerator Conferences Website pp. 1903-1905.

Variola A, Haissinski J, Loulergue A, Zomer F et al. 2014 THOMX Technical Design Report.

Wagner R F 1977 Toward a unified view of radiological imaging systems. Part II: Noisy images Medical Physics 4(4), 279-296.

Williams O, Andonian G, Babzien M, Hemsing E, Kusche K, Park J, Pogorelsky I, Priebe G, Rosenzweig J \& Yakimenko V 2009 Characterization results of the BNL ATF Compton X-ray source using K-edge absorbing foils Nuclear Instruments and Methods in Physics Research Section A: Accelerators, Spectrometers, Detectors and Associated Equipment 608(1), S18S22.

Yamada K, Kuroda R, Toyakawa H, Ikeura-Sekiguchi H, Yasumoto M, Koike M, Sakai F, Mori K, Mori $\mathrm{H}$, Fukuyama N et al. $2009 \mathrm{~A}$ trial for fine and low-dose imaging of biological specimens using quasi-monochromatic laser-Compton X-rays Nuclear Instruments and Methods in Physics Research Section A: Accelerators, Spectrometers, Detectors and Associated Equipment 608(1), S7-S10. 